2. DESIGN ANALYSIS TITLE

CABLE AMPACITY DERATING

3. DOCUMENT IDENTIFIER (Including Rev. No.)

BABBDA000-01717-0200-00088 REV 01 5 5 I-2, II-2, III-2, IV-2, V-37

(SCPB: 5. TOTAL PAGES 5

\begin{tabular}{|c|c|c|c|}
\hline & Print Name & Signature & Date \\
\hline 9. Originator & YUIRI SHANE & & $7 / 21 / 95$ \\
\hline 10. Checker & LESLIE FERNANDEZ & Turangs & $7 / 24 / 95$ \\
\hline 11. Lead Design Engineer & Robert E. Howell & Pabut S. Honwed & $7 / 24 / 95$ \\
\hline 12.QA Manager & Daniel M. Jenkins & DM tenkins & $07 \cdot 24 \cdot 95$ \\
\hline 13. Department Manager & Gene N. Kimura & snke & $7 / 2+195$ \\
\hline
\end{tabular}
14. REMARKS 
(1.) $\mathrm{QA}: \mathrm{N} / \mathrm{A}$ Page: 2 Of: 5

\section{DESIGN ANALY'SIS TITLE}

CABLE AMPACITY DERATING

3. DOCUMENT IDENTIFIER (Including Rev. No.)

4. REVISION NO.

BABBDA000-01717-0200-00088 REV 01

\begin{tabular}{|l|l|l}
\hline 5. Revision No. & 6. Total Pages & \\
\hline 00 & 5 & Initial issue \\
01 & 5 & $\begin{array}{l}\text { Removed TBV-127 } \\
\text { Reformatted entire Design Analysis to conform with Procedure QAP-3-9 Rev. } 5\end{array}$
\end{tabular}




\section{PURPOSE}

The purpose and objective of this calculation is to determine the maximum ampacity of the 1 cables in the underground duct bank for ensuring compliance with cable limits.

\section{QUALITY ASSURANCE}

2.1 No quality assurance (QA) controls apply to the items and activities as they are discussed within this analysis and it is therefore QA classified as "NONE" per [Quality Administrative Procedure] QAP-2-0 Rev. 2, QAP-2-3 Rev. 7, and [Implementing Line Procedure] NLP-3-18 Rev. 2.

2.2 No applicable Determination of Importance Evaluations (DIEs) (Reference 5.1) have resulted in controls that would affect the structures, systems, or components/configuration items described within this analysis. If a subsequent DIE indicates that the information provided in this analysis is required for use as a design input, this design analysis will require revision.

2.3 This study was revised in accordance with QAP-3-9 Rev. 5 .

\section{METHOD}

The computer program developed by Operation Technology Inc. was used for this design. This program uses the following factors to determine the cable ampacity and temperature:
A. Ambient temperature
B. Soil thermal resistivity
C. Cable position in the duct-bank

\section{DESIGN INPUTS}

\subsection{DESIGN PARAMETERS}

- Earth Thermal Resistivity - 120 RHO (NEC 93, Appendix B)

- Fill (concrete) - 85 RHO (ETAP)

- Ambient Temperature $-40^{\circ} \mathrm{C}$

- Cable temperature not to exceed $90^{\circ} \mathrm{C}$ 


\title{
4.2 CRITERIA
}

Exploratory Studies Facility Basis For Design 7.2 IV.1

\section{| 4.3 ASSUMPTIONS}

4.3.1 Typical values for DC resistance, insulation thermal resistance, and dielectric losses are assumed for each cable size.

4.3.2 Following loads are assumed for the feeders:

1. Subsurface feeder no. 1 - 25 MVA

2. Subsurface feeder no. 2 - $25 \mathrm{MVA}$

3. Tunnel Boring Machine - 4.5 MVA

4. $480 \mathrm{~V}$ transformer loads at full capacity.

\subsection{CODES AND STANDARDS}

\author{
4.4.1 NFPA 70-1993 National Electrical Code (NEC)
}

4.4.2 ANSI/IEEE 442-1981 A Guide for Soil Thermal Resistivity Measurements

\section{REFERENCES}

I 5.1 BABB00000-01717-2200-00001 REV. 05, DIE For ESF North Portal Pad

\section{USE OF COMPUTER SOFTWARE}

I The Electrical Transient Analysis Program (ETAP) by Operation Technology, Inc. (OTI) Version

I 6.52 was used for cable ampacity derating calculations. The software used was appropriate for I the nonquality affecting application.

\section{DESIGN ANALYSIS}

Cable derating program was run to determine the maximum load current capacity and cable temperature for different duct bank configuration. Earth and fill resistivity and ambient temperature were assumed as close as possible to actual environmental conditions. Each duct will contain three-single conductor copper cables. Cable sizes are shown on the computer printout. 


\section{CONCLUSION}

Cable ampacity and cable temperature were calculated for the following duct bank configuration:

1. $3 \times 8$ main duct bank (permanent power)

2. $3 \times 8$ main duct bank (temporary power)

3. $1 \times 3$ duct bank ( $480 \mathrm{~V}$ cables to pad mount transformer)

4. $2 \times 5$ duct bank (from pole to $15 \mathrm{kV}$ switchgear).

In each case above, it was determined that cable temperature and ampacities were within the cable limits.

\section{ATTACHMENTS}

Computer Printout Study Case: 1

II Computer Printout Study Case: 2

III Computer Printout Study Case: 3

IV Computer Printout Study Case: 4

$\mathrm{V}$ Support Documents 


\section{COMPUTER PRINTOUT STUDY CASE: 1}

Project" ESF DESIGH-YUCCA MOUNTAIN

Location: LAS VEGAS, NY.

Contract: 4580823

Engineer: B.MAJHOAR
ETAP 6.52

Stuoty Case: 1

$2 \times 5$ DUCT BANK (FROM POLE TO $15 \mathrm{KV}$ SWITCHGEAR)

\begin{tabular}{|c|c|c|c|c|c|c|c|c|c|}
\hline $\begin{array}{l}\text { Cable } \\
\text { size }\end{array}$ & $\begin{array}{l}\text { No of } \\
\text { Cond. }\end{array}$ & $\begin{array}{l}\text { Volt } \\
k V\end{array}$ & Type & $\begin{array}{l}\text { DC Res. } \\
\mu \mathrm{hm} / \mathrm{ft}\end{array}$ & $\begin{array}{l}0.0 . \\
\text { (in) }\end{array}$ & $\begin{array}{l}\text { Insul. } \\
\text { Ther }, R \\
\text { ohm-ft }\end{array}$ & $\begin{array}{l}\text { Dielec } \\
\text { Losses } \\
(W / f t)\end{array}$ & $Y_{C}$ & Ys \\
\hline 750 & 1 & 15 & CU & 14.40 & 1.811 & 1.549 & 0.161 & 0.038 & 0.000 \\
\hline SPR & 0 & 0 & & 0.00 & 1.000 & 0.000 & 0.000 & 0.000 & 0.000 \\
\hline
\end{tabular}

\begin{tabular}{|c|c|c|c|c|c|c|c|c|c|}
\hline \multirow[b]{2}{*}{$\begin{array}{l}\text { Instal- } \\
\text { lation }\end{array}$} & \multirow{2}{*}{$\begin{array}{l}\text { Conduit } \\
\text { Type }\end{array}$} & \multirow{2}{*}{$\begin{array}{l}\text { \# of } \\
\text { Rows }\end{array}$} & \multirow{2}{*}{$\begin{array}{l}\text { \# of } \\
\text { Cols }\end{array}$} & \multirow{2}{*}{$\begin{array}{l}\text { Ref. } \\
\text { Depth } \\
\text { (in) }\end{array}$} & \multirow{2}{*}{$\begin{array}{l}\text { Height } \\
\text { (in) }\end{array}$} & \multirow{2}{*}{$\begin{array}{r}\text { Width } \\
\text { (in) }\end{array}$} & \multicolumn{2}{|c|}{ RHO } & \multirow{2}{*}{$\begin{array}{l}\text { Ambient } \\
\text { Temp. } \\
{ }^{\circ} \mathrm{C}\end{array}$} \\
\hline & & & & & & & Earth & Fill & \\
\hline Duct Bank & PVC & 2 & 5 & 24.0 & 28.5 & 57.0 & 120.0 & 85.0 & 40.0 \\
\hline
\end{tabular}

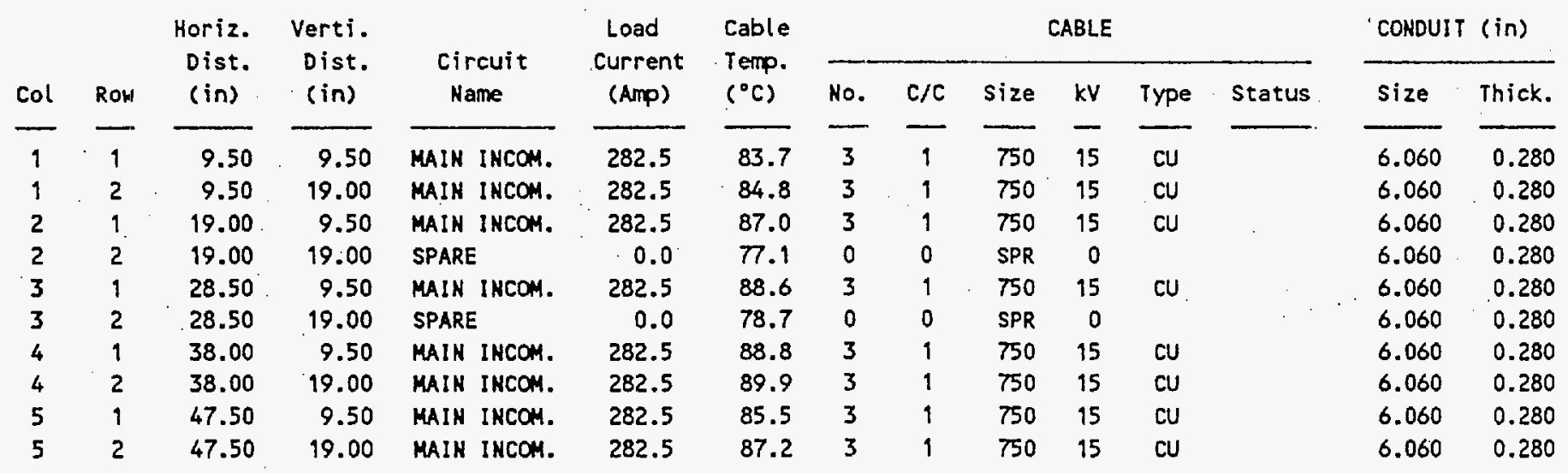




\section{COMPUTER PRINTOUT STUDY CASE: 1 (CONTINUED)}

Project: ESF DESIGN-YUCCA MOUNTAIH

Location: LAS VEGAS, NY.

Contract: 4580823

Engineer: B.MAJMUOAR
ETAP 6.52

Study Case: 1
Page: $2 / 2$

Date: $09-24-1993$

SN: $\quad 88 F D i 30132$

File: MAINDUCS

$2 \times 5$ DUCT BANK (FROA POLE TO $15 \mathrm{kV}$ SWITCHGEAR)

\begin{tabular}{|c|c|c|c|c|c|c|}
\hline & COLUANS: & 1 & 2 & 3 & 4 & 5 \\
\hline \multirow{3}{*}{ RON 1} & Cable: & 750 & 750 & 750 & 750 & 750 \\
\hline & Amp.: & 282.5 & 282.5 & 282.5 & 282.5 & 282.5 \\
\hline & Temp.: & 83.7 & 87.0 & 88.6 & 88.8 & 85.5 \\
\hline \multirow{3}{*}{ ROW 2} & Cable: & 750 & SPR & SPR & 750 & 750 \\
\hline & Amp.: & 282.5 & 0.0 & 0.0 & 282.5 & 282.5 \\
\hline & Temp.: & 84.8 & 77.1 & 78.7 & 89.9 & 87.2 \\
\hline
\end{tabular}

* Indicates locations where the calculated temperature exceeds the rated - conductor temperature as specified in the cable libraries. 


\section{COMPUTER PRINTOUT STUDY CASE: 2}

Project: ESF DESIGN-YUCCA MOUNTAIN

Location: LAS VEGAS, NV.

Contract: 4580823

Engineer: B.MAJHUOAR
ETAP 6.52

Study Case: 2
Page: $1 / 2$

Date: $09-24-1993$

SH: $88 F 0130132$

File: MAIHOUCG

$3 \times 8$ MAIN DUCT BANK (TEMPORARY PONER)

\begin{tabular}{|c|c|c|c|c|c|c|c|c|c|}
\hline Cable & $\begin{array}{l}\text { No of } \\
\text { Cond. }\end{array}$ & $\begin{array}{c}\text { Volt } \\
k V\end{array}$ & Type & $\begin{array}{l}\text { DC Res. } \\
\text { tohm/ft }\end{array}$ & $\begin{array}{l}0.0 . \\
\text { (in) }\end{array}$ & $\begin{array}{l}\text { Insul. } \\
\text { Ther.R } \\
\text { ohm-ft }\end{array}$ & $\begin{array}{l}\text { Dielec } \\
\text { Losses } \\
(H / f t)\end{array}$ & $Y_{C}$ & Ys \\
\hline SPR & 0 & 0 & & 0.00 & 1.000 & 0.000 & 0.000 & 0.000 & 0.000 \\
\hline 750 & 1 & 15 & CU & 14.40 & 1.811 & 1.549 & 0.161 & 0.038 & 0.000 \\
\hline
\end{tabular}

\begin{tabular}{|c|c|c|c|c|c|c|c|c|c|}
\hline \multirow[b]{2}{*}{$\begin{array}{l}\text { Instal- } \\
\text { lation }\end{array}$} & \multirow[b]{2}{*}{$\begin{array}{l}\text { Conduit } \\
\text { Type }\end{array}$} & \multirow[b]{2}{*}{$\begin{array}{l}\text { \# of } \\
\text { Rows }\end{array}$} & \multirow[b]{2}{*}{$\begin{array}{l}\text { \# of } \\
\text { Cols }\end{array}$} & \multirow{2}{*}{$\begin{array}{l}\text { Ref. } \\
\text { Depth } \\
\text { (in) }\end{array}$} & \multirow[b]{2}{*}{$\begin{array}{l}\text { Height } \\
\text { (in) }\end{array}$} & \multirow[b]{2}{*}{$\begin{array}{l}\text { Width } \\
\text { (in) }\end{array}$} & \multicolumn{2}{|c|}{ RHO } & \multirow{2}{*}{$\begin{array}{c}\text { Ambient } \\
\text { Temp. } \\
{ }^{\circ} \mathrm{C}\end{array}$} \\
\hline & & & & & & & Earth & Fill & \\
\hline Duct Bank & PVC & 3 & 8 & 24.0 & 38.0 & 85.5 & 120.0 & 85.0 & 40.0 \\
\hline
\end{tabular}

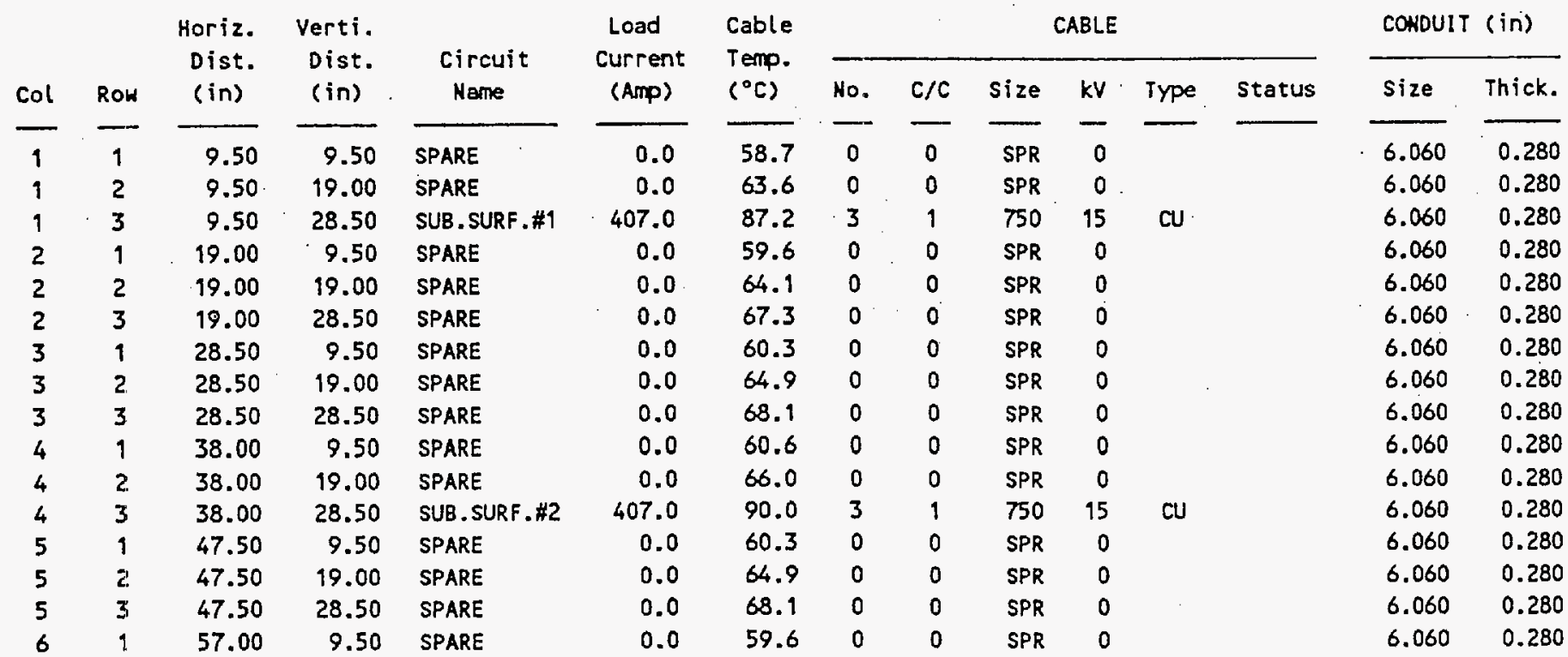




\section{COMPUTER PRINTOUT STUDY CASE: 2 (CONTINUED)}

Project: ESF DESIGN-YUCCA MOUHTAIN

Location: LAS VEGAS, NV.

Contract: 4580823

Engineer: B.MAJHDOAR PAD
ETAP 6.52

Study Case: 2
Page: $2 / 2$

Date: $09-24-1993$

SN: $88 \mathrm{FDI} 130132$

File: MAINDUC6

\begin{tabular}{|c|c|c|c|c|c|c|c|c|c|c|c|c|c|c|}
\hline \multicolumn{2}{|r|}{ ROH } & $\begin{array}{c}\text { Horiz. } \\
\text { Dist. } \\
\text { (in) }\end{array}$ & $\begin{array}{c}\text { Verti. } \\
\text { Dist. } \\
\text { (in) }\end{array}$ & $\begin{array}{l}\text { Circuit } \\
\text { Name }\end{array}$ & $\begin{array}{l}\text { Load } \\
\text { Current } \\
\text { (Amp) }\end{array}$ & $\begin{array}{l}\text { Cable } \\
\text { Temp. } \\
\left({ }^{\circ} \mathrm{C}\right)\end{array}$ & \multicolumn{6}{|c|}{ CABLE } & \multicolumn{2}{|c|}{ CONDUIT (in) } \\
\hline 7 & 2 & 66.50 & 19.00 & SPARE & 0.0 & 63.6 & 0 & 0 & SPR & 0 & & & 6.060 & 0.280 \\
\hline 7 & 3 & 66.50 & 28.50 & TBM LOAD & 407.0 & 87.2 & 3 & 1 & 750 & 15 & $\mathrm{CU}$ & & 6.060 & 0.280 \\
\hline 8 & 1 & 76.00 & 9.50 & SPARE & 0.0 & 57.1 & 0 & 0 & SPR & 0 & & & 6.060 & 0.280 \\
\hline
\end{tabular}

\begin{tabular}{|c|c|c|c|c|c|c|c|c|c|}
\hline & COLLANS: & 1 & 2 & 3 & 4 & 5 & 6 & 7 & 8 \\
\hline \multirow{3}{*}{ ROH 1} & Cable: & SPR & SPR & SPR & SPR & SPR & SPR & SPR & SPR \\
\hline & Amf.: : & 0.0 & 0.0 & 0.0 & 0.0 & 0.0 & 0.0 & 0.0 & 0.0 \\
\hline & Temp.: & 58.7 & 59.6 & 60.3 & 60.6 & 60.3 & 59.6 & 58.7 & 57.1 \\
\hline \multirow{3}{*}{ ROW 2} & Cable: & SPR & SPR & SPR & SPR & SPR & SPR & SPR & SPR \\
\hline & Amp.: : & 0.0 & 0.0 & 0.0 & 0.0 & 0.0 & 0.0 & 0.0 & 0.0 \\
\hline & Templ.: & 63.6 & 64.1 & 64.9 & 66.0 & 64.9 & 64.1 & 63.6 & 60.6 \\
\hline \multirow{3}{*}{ RON 3} & Cable: & 750 & SPR & SPR & 750 & SPR & SPR & 750 & SPR \\
\hline & Anfo.: & 407.0 & 0.0 & 0.0 & 407.0 & 0.0 & 0.0 & 407.0 & 0.0 \\
\hline & Temp.: & 87.2 & 67.3 & 68.1 & 90.0 & 68.1 & 67.3 & 87.2 & 63.3 \\
\hline
\end{tabular}


COMPUTER PRINTOUT STUDY CASE: 3

Project: ESF DESIGN-YUCCA MOUNTAIN

Location: LAS VEGAS, NV.

Contract: 4580823

Engineer: B.MAJMDAR PA
ETAP 6.52

Stucty Case: 3
Page: $1 / 2$

Date: $09-24-1993$

SN: $\quad$ Q8FDI30132

File: MAINDUC7

$3 \times 8$ MAIN DUCT BAMK (PERMANENT POWER)

\begin{tabular}{|c|c|c|c|c|c|c|c|c|c|}
\hline $\begin{array}{l}\text { Cable } \\
\text { Size }\end{array}$ & $\begin{array}{l}\text { No of } \\
\text { Cond. }\end{array}$ & $\begin{array}{l}\text { Volt } \\
k V .\end{array}$ & Type & $\begin{array}{l}\text { DC Res. } \\
\text { Hohm/ft }\end{array}$ & $\begin{array}{l}0.0 . \\
\text { (in) }\end{array}$ & $\begin{array}{l}\text { Insul. } \\
\text { Ther.R } \\
\text { ohm-ft }\end{array}$ & $\begin{array}{l}\text { Dielec } \\
\text { Losses } \\
(H / f t)\end{array}$ & $Y c$ & Ys \\
\hline 750 & 1 & 15 & CU & 14.40 & 1.811 & 1.549 & 0.161 & 0.038 & 0.000 \\
\hline $4 / 0$ & 1 & 15 & CU & 50.90 & 1.315 & 2.353 & 0.100 & 0.004 & 0.000 \\
\hline SPR & 0 & 0 & & 0.00 & 1.000 & 0.000 & 0.000 & 0.000 & 0.000 \\
\hline 500 & 1 & 15 & $\mathrm{CU}$ & 21.60 & 1.597 & 1.752 & 0.137 & 0.016 & 0.000 \\
\hline
\end{tabular}

\begin{tabular}{|c|c|c|c|c|c|c|c|c|c|}
\hline \multirow{2}{*}{$\begin{array}{l}\text { Instal- } \\
\text { lation. }\end{array}$} & \multirow{2}{*}{$\begin{array}{c}\text { Conduit } \\
\text { Type }\end{array}$} & \multirow{2}{*}{$\begin{array}{l}\text { \# of } \\
\text { Rows }\end{array}$} & \multirow{2}{*}{$\begin{array}{l}\text { \# of } \\
\text { Cols }\end{array}$} & \multirow{2}{*}{$\begin{array}{l}\text { Ref. } \\
\text { Depth } \\
\text { (in) }\end{array}$} & \multirow{2}{*}{$\begin{array}{l}\text { Height } \\
\text { (in) }\end{array}$} & \multirow{2}{*}{$\begin{array}{l}\text { Width } \\
\text { (in) }\end{array}$} & \multicolumn{2}{|c|}{ RHO } & \multirow{2}{*}{$\begin{array}{c}\text { Ambient } \\
\text { Temp. } \\
{ }^{\circ} \mathrm{C}\end{array}$} \\
\hline & & & & & & & Earth & Fill & \\
\hline Duct Bank & PVC & 3 & 8 & 24.0 & 38.0 & 85.5. & 120.0 & 85.0 & 40.0 \\
\hline
\end{tabular}

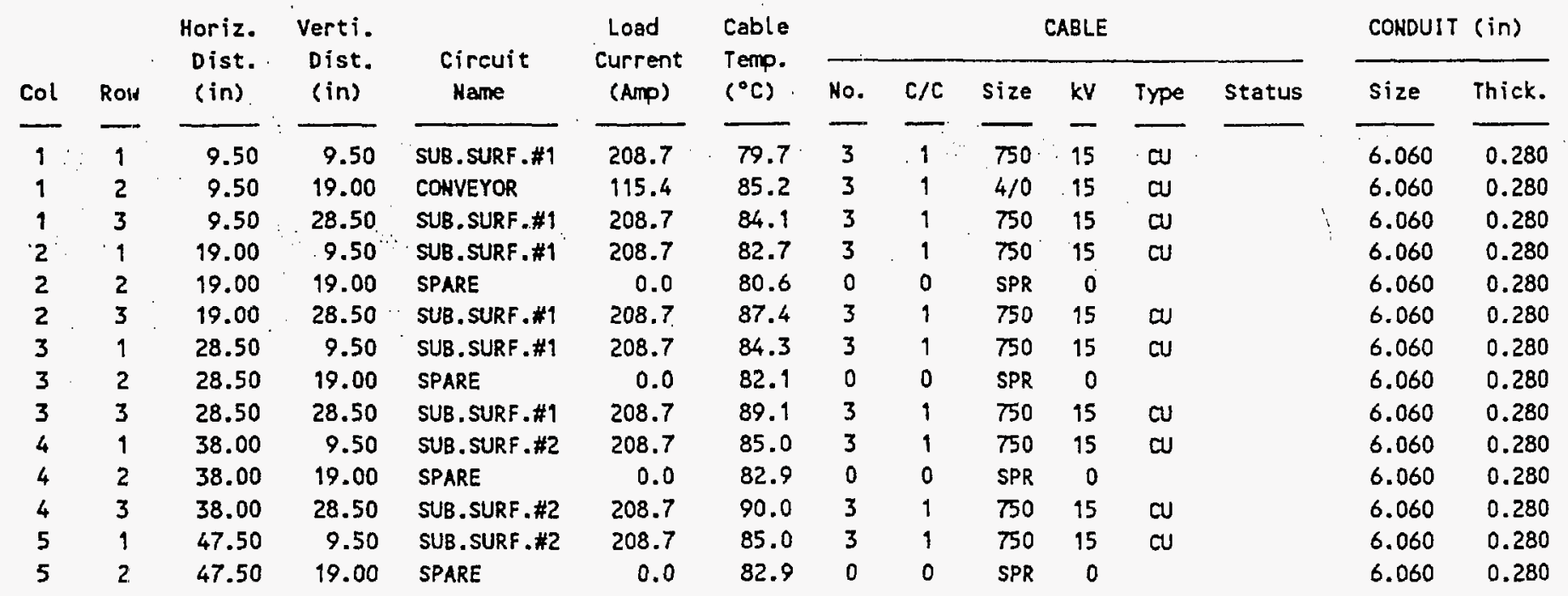




\section{COMPUTER PRINTOUT STUDY CASE: 3 (CONTINUED)}

Project: ESF DESIGK-YUCCA HOUNTAIN Location: LAS VEGAS, NV.

Contract: 4580823

Engineer: B.MAJMUOAR
ETAP 6.52

Study Case: 3
Page: $2 / 2$

Date: $09-24-1993$

SN: $\quad 88 F D 130132$

File: MAINDUC7

$3 \times 8$ MAIN DUCT BAKK (PERMANENT POWER)

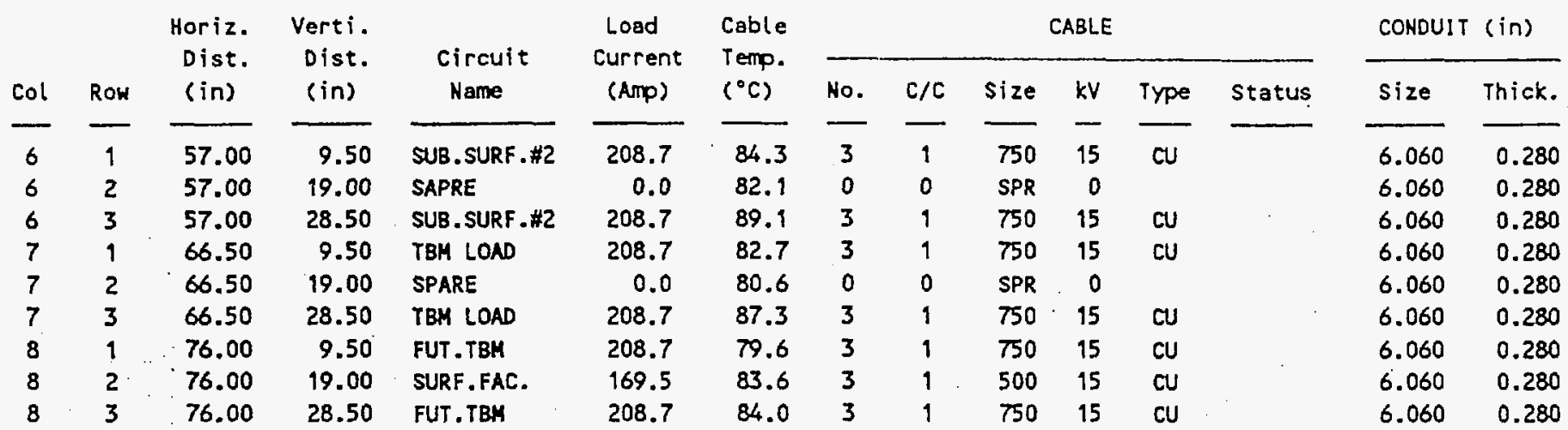

\begin{tabular}{|c|c|c|c|c|c|c|c|c|c|}
\hline & COLUMNS: & 1 & 2 & 3 & 4 & 5 & 6. & 7 & 8 \\
\hline . & Cable: & 750 & 750 & 750 & 750 & 750 & 750 & 750 & 750 \\
\hline \multirow[t]{3}{*}{ ROW 1} & Amp.: & 208.7 & 208.7 & 208.7 & 208.7 & 208.7 & 208.7 & 208.7 & 208.7 \\
\hline & Temp.: & 79.7 & 82.7 & 84.3 & 85.0 & 85.0 & 84.3 & 82.7 & 79.6 \\
\hline & Cable: & $4 / 0$ & SPR & SPR & SPR & SPR & SPR & SPR & 500 \\
\hline \multirow[t]{3}{*}{ ROW 2} & Amp.: & 115.4 & 0.0 & 0.0 & 0.0 & 0.0 & 0.0 & 0.0 & 169.5 \\
\hline & Temp.: & 85.2 & 80.6 & 82.1 & 82.9 & 82.9 & 82.1 & 80.6 & 83.6 \\
\hline & Cable: & 750 & 750 & 750 & 750 & 750 & 750 & 750 & 750 \\
\hline \multirow[t]{2}{*}{ ROW 3} & Amp.: & 208.7 & 208.7 & 208.7 & 208.7 & 208.7 & 208.7 & 208.7 & 208.7 \\
\hline & Temp.: & 84.1 & 87.4 & 89.1 & 90.0 & 89.9 & 89.1 & 87.3 & 84.0 \\
\hline
\end{tabular}

- Indicates locations where the calculated temperature exceeds the rated conductor temperature as specified in the cable libraries. 
COMPUTER PRINTOUT STUDY CASE: 4

Project: ESF DESIGN-YUCCA HOUNTAIH

Location: LAS VEGAS, NV.

Contract: 4580823

Engineer: B.MAJMUDAR $\beta A-$
ETAP 6.52

Study Case: 4
Page: $1 / 2$

Date: 09-24-1993

SN: $88 F D 130132$

File: 480 VCuc3

$1 \times 3$ DUCT BANK ( $480 \mathrm{~V}$ CABLES TO PAD MOUNT TRAKSFORHER)

\begin{tabular}{|c|c|c|c|c|c|c|c|c|c|}
\hline $\begin{array}{l}\text { Cable } \\
\text { size }\end{array}$ & $\begin{array}{l}\text { No of } \\
\text { Cond. }\end{array}$ & $\begin{array}{c}\text { Volt } \\
\mathrm{kV}\end{array}$ & Type & $\begin{array}{l}\text { DC Res. } \\
\mu \circ h m / f t\end{array}$ & $\begin{array}{l}\text { O. D. } \\
\text { (in) }\end{array}$ & $\begin{array}{l}\text { Insul. } \\
\text { Ther.R } \\
\text { ohm-ft }\end{array}$ & $\begin{array}{l}\text { Dielec } \\
\text { Losses } \\
(\mathrm{H} / \mathrm{ft})\end{array}$ & Yc & Ys \\
\hline 500 & 1 & 1 & Cu & 21.60 & 1.189 & 0.983 & 0.000 & 0.016 & 0.000 \\
\hline
\end{tabular}

\begin{tabular}{|c|c|c|c|c|c|c|c|c|c|}
\hline \multirow{2}{*}{$\begin{array}{l}\text { Instal- } \\
\text { lation }\end{array}$} & \multirow{2}{*}{$\begin{array}{l}\text { Conduit } \\
\text { Type }\end{array}$} & \multirow{2}{*}{$\begin{array}{l}\text { \# of } \\
\text { Rows }\end{array}$} & \multirow{2}{*}{$\begin{array}{l}\text { \# of } \\
\text { Cols }\end{array}$} & \multirow{2}{*}{$\begin{array}{l}\text { Ref. } \\
\text { Depth } \\
\text { (in) }\end{array}$} & \multirow{2}{*}{$\begin{array}{c}\text { Height } \\
\text { (in) }\end{array}$} & \multirow{2}{*}{$\begin{array}{l}\text { Width } \\
\text { (in) }\end{array}$} & \multicolumn{2}{|c|}{ RHO } & \multirow{2}{*}{$\begin{array}{c}\text { Ambient } \\
\text { Temp. } \\
{ }^{\circ} \mathrm{C}\end{array}$} \\
\hline & & & & & & & Earth & Fill & \\
\hline Duct Bank & PVC & 1 & 3 & 24.0 & 17.0 & 34.0 & 120.0 & 85.0 & 40.0 \\
\hline
\end{tabular}

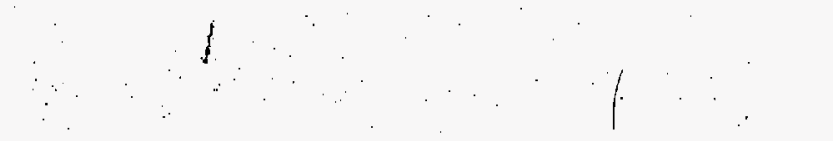

\begin{tabular}{|c|c|c|c|c|c|c|c|c|c|c|c|c|c|c|}
\hline \multirow[b]{2}{*}{ Col } & \multirow[b]{2}{*}{ Ron } & \multirow{2}{*}{$\begin{array}{c}\text { Horiz. } \\
\text { Dist. } \\
\text { (in) }\end{array}$} & \multirow{2}{*}{$\begin{array}{l}\text { Verti. } \\
\text { Dist. } \\
\text { (in) }\end{array}$} & \multirow{2}{*}{$\begin{array}{c}\text { Circuit } \\
\text { Name }\end{array}$} & \multirow{2}{*}{$\begin{array}{l}\text { Load } \\
\text { Current } \\
\text { (Amp) }\end{array}$} & \multirow{2}{*}{$\begin{array}{l}\text { Cable } \\
\text { Temp. } \\
\left({ }^{\circ} \mathrm{C}\right)\end{array}$} & \multicolumn{6}{|c|}{ CABLE } & \multicolumn{2}{|c|}{ CONDUIT (in) } \\
\hline & & & & & & & No. & $C / C$ & size & $k V$ & Type & status & size & Thick. \\
\hline 1 & 1 & 8.50 & 8.50 & $A 1$ & 310.2 & 87.5 & 3 & 1 & 500 & 1 & CU & & 5.040 & 0.260 \\
\hline 2 & 1 & 17.00 & 8.50 & B1 & 310.2 & 90.0 & 3 & 1 & 500 & $\mathfrak{i}$ & $\mathrm{CU}$ & & 5.040 & 0.260 \\
\hline 3 & 1 & 25.50 & 8.50 & c1 & 310.2 & 87.5 & 3 & 1 & 500 & .1 & CU & & 5.040 & 0.260 \\
\hline
\end{tabular}


COMPUTER PRINTOUT STUDY CASE: 4 (CONTINUED)

Project: ESF DESIGH-YUCCA MOUNTAIH

ETAP 6.52

Page: IV-2

Location: LAS VEGAS, NV.

Contract: 4580823

Engineer: B.MAJMLOAR $P^{2}$

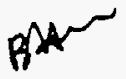

Study Case: 4
Page: $2 / 2$
Date: $09-24-1993$
SN: $88 F D 130132$
File: 480vcuc3

$1 \times 3$ DUCT BANK (48OV CABLES TO PAD MOUNT TRANSFORMER)

\begin{tabular}{|c|c|c|c|c|}
\hline & COLUMNS: & 1 & 2 & 3 \\
\hline \multirow{3}{*}{ RON 1} & Cable: & 500 & 500 & 500 \\
\hline & Anp.: & 310.2 & 310.2 & 310.2 \\
\hline & Temp.: & 87.5 & 90.0 & 87.5 \\
\hline
\end{tabular}

* Indicates-locations where the calculated temperature exceeds the rated conductor temperature as specified in the cable libraries. 
ATTACHMENT V

DI: BABBDA000-01717-0200-00088 Rev. 01 I

Title: Cable Ampacity Derating

Page: V-1

SUPPORT DOCUMENTS

$15 \mathrm{KV} / \mathrm{C}$ SHCD EPR/PVC $133 \%$ inSUL. $750 \mathrm{MCM} \quad D 1 A=1.89 \quad 2.8$

$500 \mathrm{mcm}$

$1.55 \quad 1.89$

$5^{\prime \prime}$ CONOUIT $5.047=20.0^{\prime \prime}$ AnEA $53 \%=10.6^{\prime \prime} \quad 40 \%=8^{\prime \prime} 31 \%=6.2$

6 "CONDUIT 6.065 = 28.89 AMAA 53\% $=15.3140 \%=11.56$ " $31 \%=8.96$

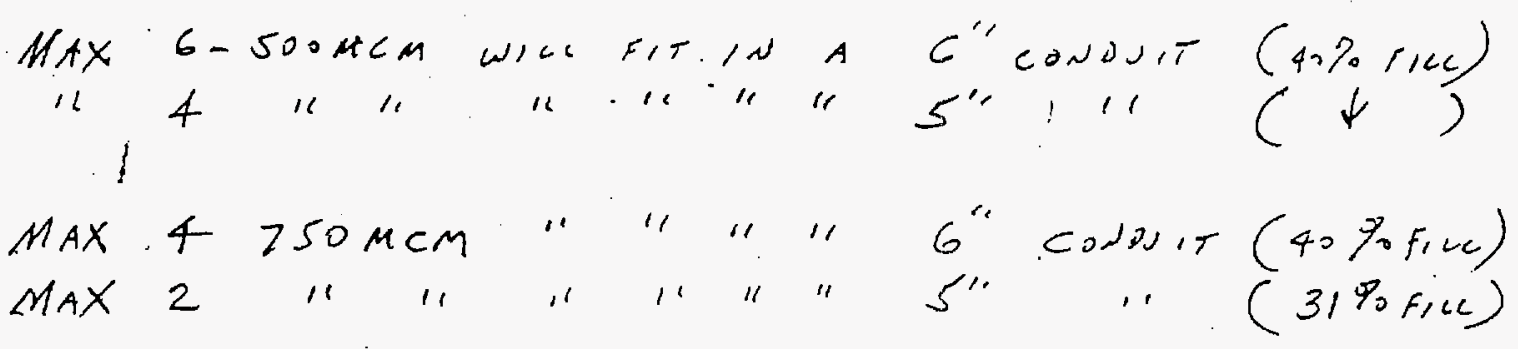




\section{SUPPORT DOCUMENTS (CONTINUED)}

\section{CABLE DERATING AND DUCT BANK LAYOUT}

STUDY CASE \#1

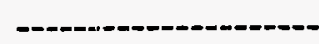

STUDY CASE \#2

STUDY CASE \#3

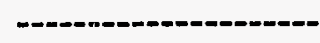

$3 / \mathrm{C} \# 750 \mathrm{kcmil}$ (For tunnel load)

1160A / 205.7 = 6 Cables Per Phase

$(1-3 / \mathrm{C} \# 750 \mathrm{kcmil}$ in a duct $)$

$1 / \mathrm{C} \# 500 \mathrm{kcmil}$ (For tunnel load)

1160A $/ 175.9=7$ Cable Per Phase

(3-1/C \#500 kcmil in a duct)

$1 / \mathrm{C} \# 750 \mathrm{kcmil}$ (For tunnel load)

1160A $/ 211.7=6$ Cable Per Phase

(3-1/C \#750 $\mathrm{kcmil}$ in a duct)

Page: V-2

DUCT CONFIGURATION

\begin{tabular}{|c|c|c|c|}
\hline DESCRIPTION & STUDY CASE \#1 & STUDY CASE \#2 & STUDY CASE \# 3 \\
\hline TUNN.1A LOAD & 6 Ducts & 7 Ducts & 6 Ducts \\
\hline TUNN.1B LOAD & 6 Ducts & 7 Ducts & 6 Ducts \\
\hline TBM FDR. & 2 Ducts & 2 Ducts & 2 Ducts \\
\hline FUTURE TBM & 2 Ducts & 2 Ducts & 2 Ducts \\
\hline CONVEYOR & 1 Duct & 1 Duct & 1 Duct \\
\hline SURF.FAC. & 1 Duct & 1 Duct & 1 Duct \\
\hline SPARE & 6 Ducts & 7 Ducts & 6 Ducts \\
\hline TOTAL & 24 Ducts & 27 Ducts & 24 Ducts \\
\hline
\end{tabular}




\section{SUPPORT DOCUMENTS (CONTINUED)}

CABLE AMPACITY DERATING

Project: ESF DESIGN-YUCCA MOUWTAIH

Location: LAS VEGAS, NV.

Contract: 4580823

Engineer: B.MAJMUDAR

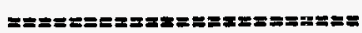

$$
\text { ETAP } 6.52
$$

Page: 1

Date: $06-17-1993$

SH: $\quad 88 F D 130132$

File: MAINDUC5

$3 \times 11$ main duct bakK $\left(750 \mathrm{kCmil}, 3 / \mathrm{C}, 9.5^{1}\right.$ SPACING, $40^{\circ} \mathrm{C}$ AMB.

\begin{tabular}{|c|c|c|c|c|c|c|c|c|c|}
\hline & & & & & & Insul. & Dielec & & \\
\hline $\begin{array}{l}\text { Cable } \\
\text { Size }\end{array}$ & $\begin{array}{l}\text { No of } \\
\text { Cond. }\end{array}$ & $\begin{array}{c}\text { Volt } \\
k V\end{array}$ & Type & $\begin{array}{l}\text { DC Res. } \\
\text { lohm/ft }\end{array}$ & $\begin{array}{l}0.0 . \\
\text { (in) }\end{array}$ & $\begin{array}{l}\text { Ther } R \\
\text { ohm-ft }\end{array}$ & $\begin{array}{l}\text { Losses } \\
(H / f t)\end{array}$ & $Y_{c}$ & Ys \\
\hline$\ldots$ & $\ldots$ & $\ldots$ & $\ldots$ & 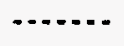 & $\ldots$ & - & - & $\ldots$ & $\ldots$ \\
\hline$\pi 0$ & 3 & 15 & CU & 14.40 & 3.790 & 1.795 & 0.160 & 0.093 & 0.000 \\
\hline SPR & 0 & 0 & & 0.00 & 1.000 & 0.000 & 0.000 & 0.000 & 0.000 \\
\hline
\end{tabular}

\begin{tabular}{|c|c|c|c|c|c|c|c|c|c|}
\hline \multirow{3}{*}{$\begin{array}{l}\text { Instal - } \\
\text { lation }\end{array}$} & \multirow{3}{*}{$\begin{array}{l}\text { Conduit } \\
\text { Type }\end{array}$} & \multirow{3}{*}{$\begin{array}{l}\text { \# of } \\
\text { Rows }\end{array}$} & \multirow{3}{*}{$\begin{array}{l}\# \text { of } \\
\text { Cols }\end{array}$} & \multicolumn{3}{|l|}{ Ref. } & \multicolumn{2}{|c|}{ RHO } & \multirow{3}{*}{$\begin{array}{l}\text { Ambient } \\
\text { Temp. } \\
\text { thc }\end{array}$} \\
\hline & & & & Depth & Height & Width & $\cdots$ & $\ldots$ & \\
\hline & & & & (in) & (in) & (in) & Earth & Fill & \\
\hline 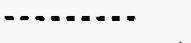 & 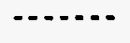 & $\cdots$ & $\cdots$ & $\ldots$ & - & -.... & $\cdots$ & $\cdots$ & $-\ldots$ \\
\hline Duct Bank: & PVC & 3 & 11 & 24.0 & 38.0 & 114.0 & 120.0 & 85.0 & 40.0 \\
\hline
\end{tabular}

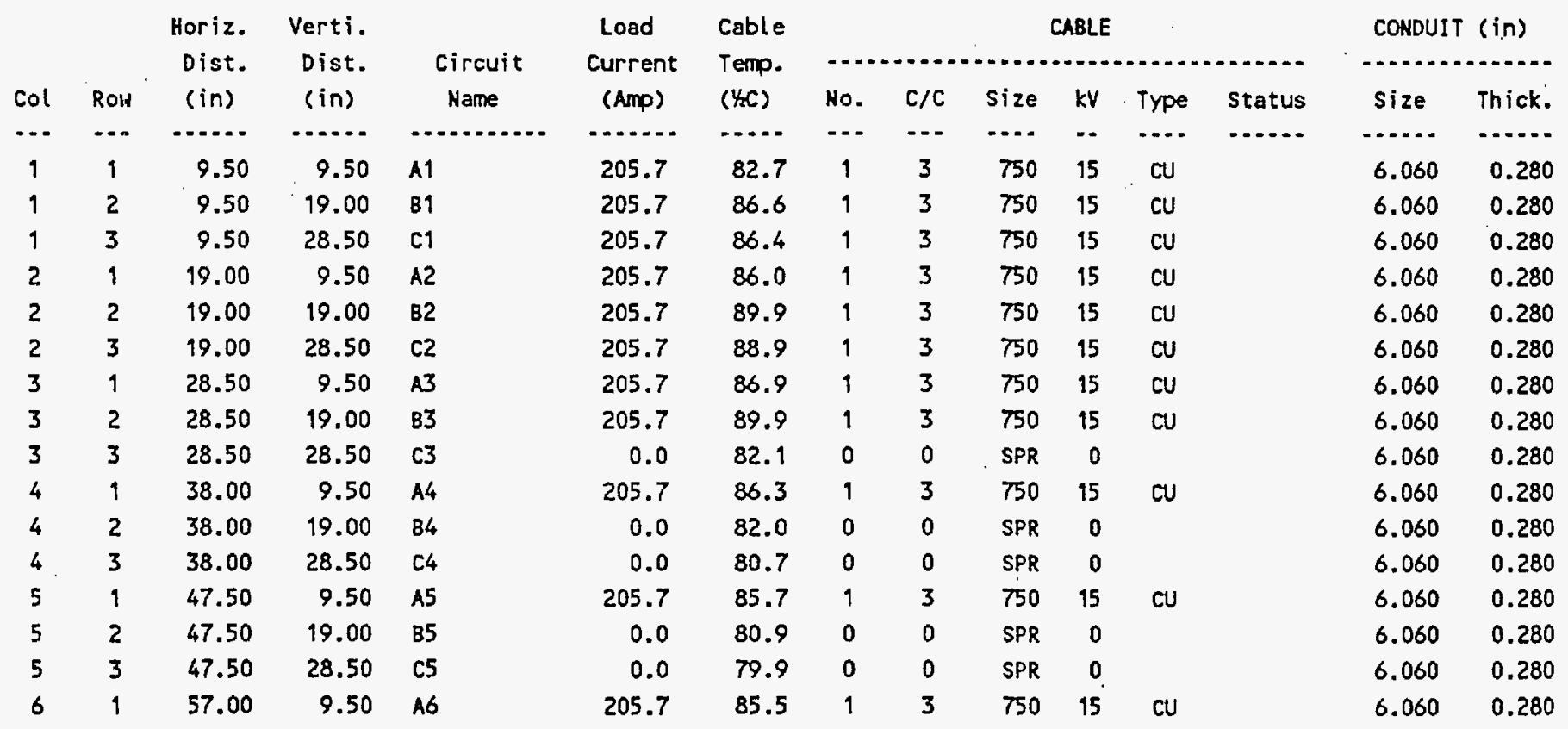




\section{SUPPORT DOCUMENTS (CONTINUED)}

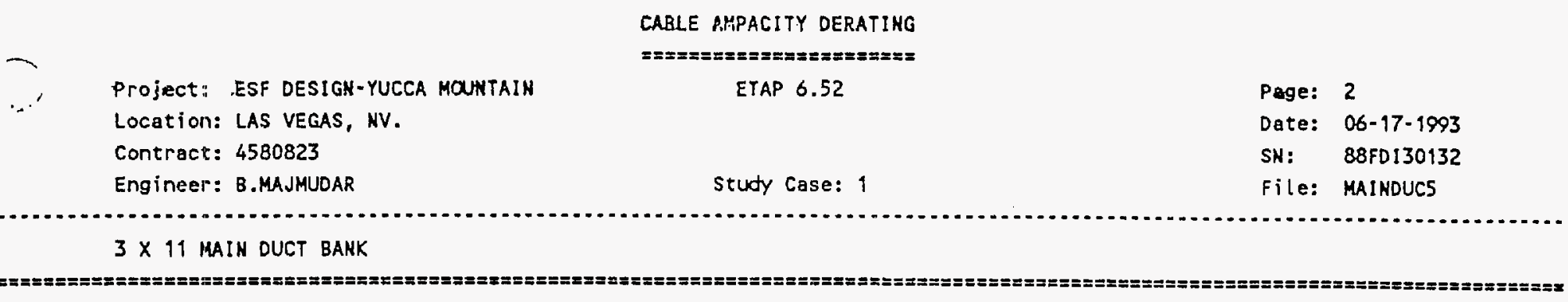

\begin{tabular}{|c|c|c|c|c|c|c|c|c|c|c|c|c|c|c|}
\hline \multirow[b]{2}{*}{ Col } & \multirow[b]{2}{*}{ Row } & \multirow{2}{*}{$\begin{array}{c}\text { Horiz. } \\
\text { Dist. } \\
\text { (in) }\end{array}$} & \multirow{2}{*}{$\begin{array}{c}\text { Verti. } \\
\text { Dist. } \\
\text { (in) }\end{array}$} & \multirow{2}{*}{$\begin{array}{l}\text { Circuit } \\
\text { Name }\end{array}$} & \multirow{2}{*}{$\begin{array}{l}\text { Load } \\
\text { Current } \\
\text { (Amp) }\end{array}$} & \multirow{2}{*}{$\begin{array}{l}\text { Cable } \\
\text { Temp. } \\
\left(k_{c} c\right)\end{array}$} & \multicolumn{6}{|c|}{ CABLE } & \multicolumn{2}{|c|}{ CONDUIT (in) } \\
\hline & & & & & & & No. & $C / C$ & Size & kV & Type & Status & size & Thick. \\
\hline$\cdots$ & $\ldots$ & ....... & $\cdots$ & - & $\ldots \ldots$ & $\cdots$ & $\cdots$ & $\cdots$ & $\cdots$ & $-\cdot$ & $\cdots$ & $\cdots+\cdots$ & $\ldots$ & $\ldots \ldots$ \\
\hline 7 & .1 & 66.50 & 9.50 & A7 & 205.7 & 85.7 & 1 & 3 & $\sqrt{50}$ & 15 & CU & & 6.060 & 0.280 \\
\hline 7 & 2 & 66.50 & 19.00 & B7 & 0.0 & 80.9 & 0 & 0 & SPR & 0 & & & 6.060 & 0.280 \\
\hline 7 & 3 & 66.50 & 28.50 & $c 7$ & 0.0 & 79.9 & 0 & 0 & SPR & 0 & & & 6.060 & 0.280 \\
\hline 8 & 1 & 76.00 & 9.50 & A8 & 205.7 & 86.3 & 1 & 3 & 750 & 15 & CU & & 6.060 & 0.280 \\
\hline 8 & 2 & 76.00 & 19.00 & B8 & 0.0 & 82.0 & 0 & 0 & SPR & 0 & & & 6.060 & 0.280 \\
\hline 8 & 3 & 76.00 & 28.50 & C8 & 0.0 & 80.7 & 0 & 0 & SPR & 0 & & & 6.060 & 0.280 \\
\hline 9 & 1 & 85.50 & 9.50 & A9 & 205.7 & 86.9 & 1 & 3 & $\sqrt{50}$ & 15 & $\mathrm{CU}$ & & 6.060 & 0.280 \\
\hline 9 & 2 & 85.50 & 19.00 & B9 & 205.7 & 89.9 & 1 & 3 & 750 & 15 & CU & & 6.060 & 0.280 \\
\hline 9 & 3 & 85.50 & 28.50 & $\mathrm{CP}$ & 0.0 & 82.1 & 0 & 0 & SPR & 0 & & & 6.060 & 0.280 \\
\hline 10 & 1 & 95.00 & 9.50 & A10 & 205.7 & 86.0 & 1 & 3 & 750 & 15 & CU & & 6.060 & 0.280 \\
\hline 10 & 2 & 95.00 & 19.00 & B10 & 205.7 & 89.9 & 1 & 3 & 750 & 15 & CU & & 6.060 & 0.280 \\
\hline 10 & 3 & 95.00 & 28.50 & C10 & 205.7 & 88.9 & 1 & 3 & 750 & 15 & $C U$ & & 6.060 & 0.280 \\
\hline 11 & 1 & 104.50 & 9.50 & A11 & 205.7 & 82.7 & 1 & 3 & 750 & 15 & CU & & 6.060 & 0.280 \\
\hline 11 & 2 & 104.50 & 19.00 & B11 & 205.7 & 86.6 & 1 & 3 & 750 & 15 & CU & & 6.060 & 0.280 \\
\hline 11 & 3 & 104.50 & 28.50 & C11 & 205.7 & 86.4 & 1 & 3 & 750 & 15 & $\mathrm{CJ}$ & & 6.060 & 0.280 \\
\hline
\end{tabular}




\section{SUPPORT DOCUMENTS (CONTINUED)}

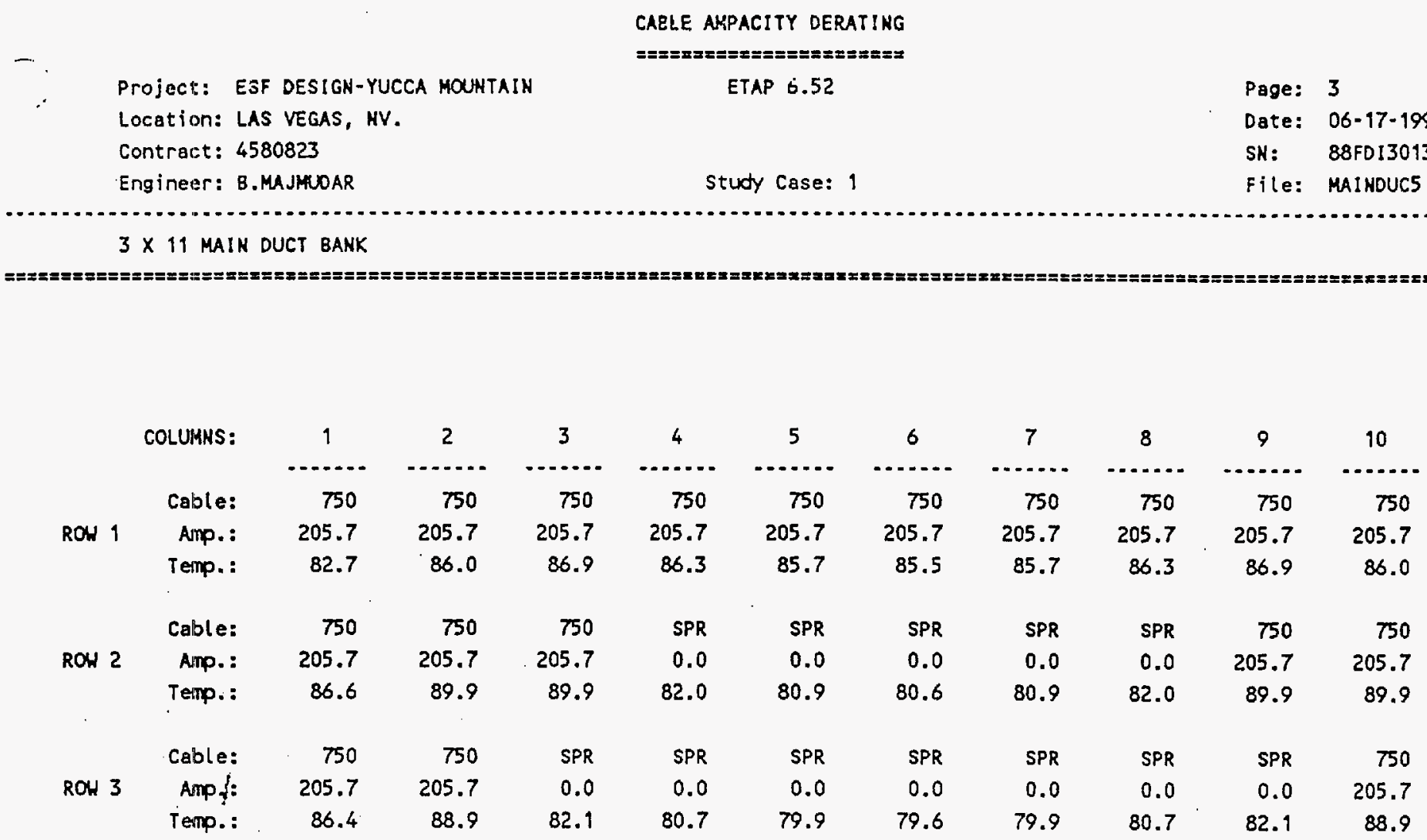

\begin{tabular}{|c|c|}
\hline COLUMNS: & 11 \\
\hline Cable: & 750 \\
\hline Amp.: & 205.7 \\
\hline Tenp.: & 82.7 \\
\hline Cable: & 750 \\
\hline Amp.: & 205.7 \\
\hline Temp.: & 86.6 \\
\hline Cable: & 750 \\
\hline Amp.: & 205.7 \\
\hline Temp.: & 86.4 \\
\hline
\end{tabular}

* Indicates locations where the calculated temperature exceeds the rated conductor temperature as specified in the cable libraries. 


\title{
SUPPORT DOCUMENTS (CONTINUED)
}

\author{
CABLE AHPACITY DERATIHG

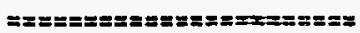 \\ ETAP 6.52 \\ Project: ESF DESIGN-YUCCA MOUNTAIN \\ Location: LAS VEGAS, KV. \\ Contract: 4580823 \\ Engineer: B.MAJMUDAR \\ Study Case: 2 \\ Parge: 1 \\ Date: $06-17-1993$ \\ SN: $88 \mathrm{FO} 130132$ \\ File: MAINDUCI
}

Page: V-6

$3 \times 11$ MAIN DUCT BANK (5OD KCmi), $1 / C, 9.5$ SPACING,40CAMB.)

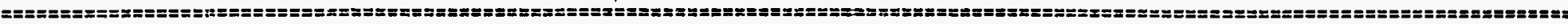

\begin{tabular}{|c|c|c|c|c|c|c|c|c|c|}
\hline $\begin{array}{l}\text { Cable } \\
\text { size }\end{array}$ & $\begin{array}{l}\text { No of } \\
\text { Cond. }\end{array}$ & $\begin{array}{c}\text { Volt } \\
k V\end{array}$ & Type & $\begin{array}{l}\text { DC Res. } \\
\text { I ohm/ft }\end{array}$ & $\begin{array}{l}0.0 . \\
\text { (in) }\end{array}$ & $\begin{array}{l}\text { Insul. } \\
\text { Ther. R } \\
\text { oheft }\end{array}$ & $\begin{array}{l}\text { Dielec } \\
\text { Losses } \\
(W / f t)\end{array}$ & Yc & Ys \\
\hline$\ldots$. & $\ldots$ & $\cdots$ & $\ldots$ & . & $\ldots$ & $\ldots \ldots$ & $\ldots$ & $\cdots$ & $\ldots$ \\
\hline 500 & 1 & 15 & $C U$ & 21.60 & 1.597 & 1.752 & 0.137 & 0.016 & 0.000 \\
\hline SPR & 1 & 0 & & 0.00 & 1.000 & 0.000 & 0.000 & 0.000 & 0.000 \\
\hline
\end{tabular}

\begin{tabular}{|c|c|c|c|c|c|c|c|c|c|}
\hline Instal:- & Conduit & \# of & \# of & $\begin{array}{l}\text { Ref. } \\
\text { Depth }\end{array}$ & Height & Width & \multicolumn{2}{|c|}{ RHO } & $\begin{array}{c}\text { Ambient } \\
\text { Temp. }\end{array}$ \\
\hline lation & Type & ROWs & cols & (in) & (in) & (in) & Earth & Fill & $\% c$ \\
\hline , n & $\ldots$ & $\cdots$ & $\cdots$ & $\cdots+$ & 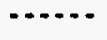 & - . . & $\cdots+$ & $\cdots$ & $\cdots$ \\
\hline Duct Bank & PVC & 3 & 11 & 24.0 & 38.0 & 114.0 & 120.0 & 85.0 & 40.0 \\
\hline
\end{tabular}

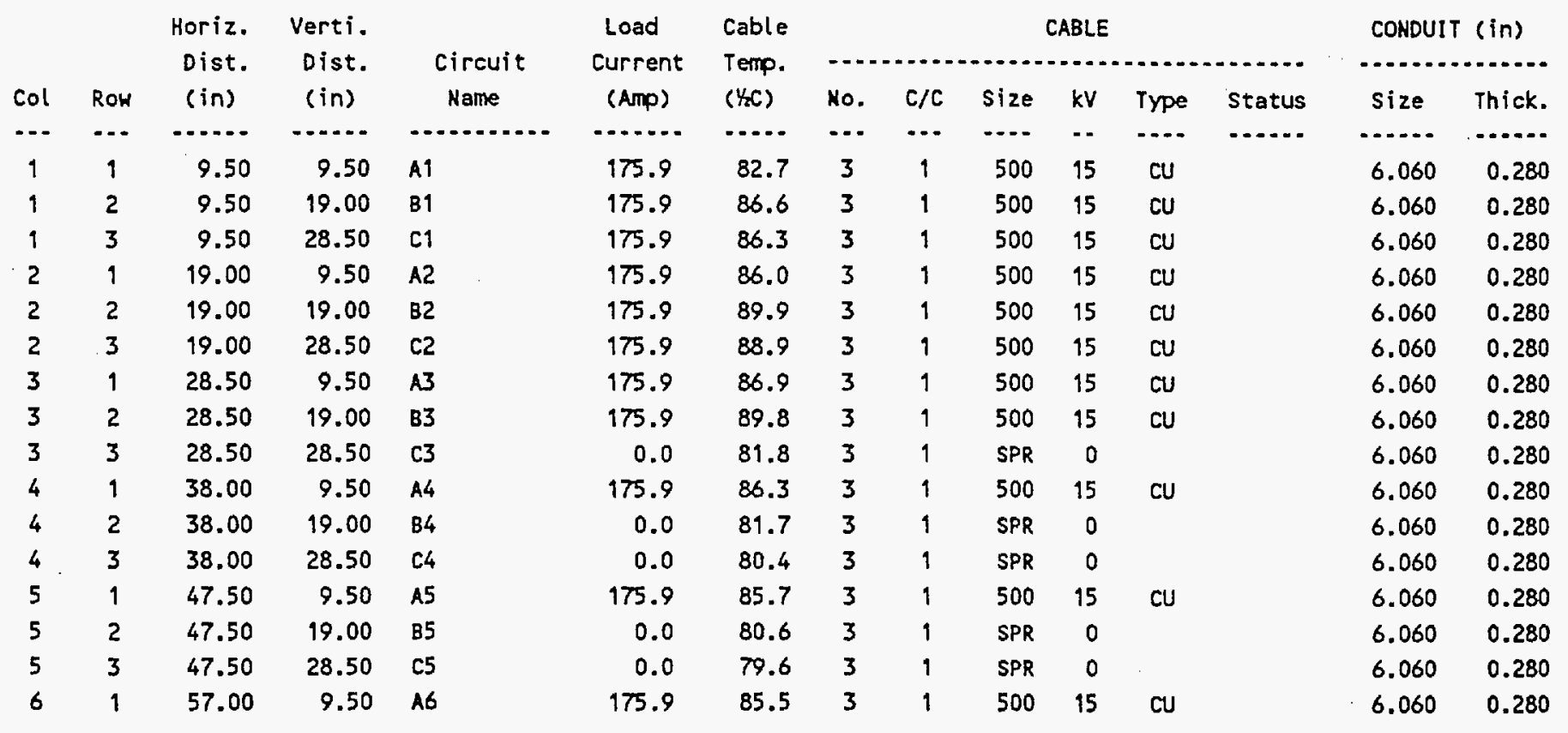




\section{SUPPORT DOCUMENTS (CONTINUED)}

\begin{tabular}{|c|c|c|c|c|}
\hline$\cdots$ & & $\begin{array}{l}\text { CABLE AMPACITY DERATING } \\
======m==========1==\end{array}$ & & \\
\hline & Project: ESF DESIGR-YUCCA MOUNTAIN & ETAP 6.52 & Page: & 2 \\
\hline & Location: LAS VEGAS, NV. & & Date: & $06-17-1993$ \\
\hline & Contract: 4580823 & & SN: & 88FDI30132 \\
\hline & Engineer: B.MAJMUOAR & Study Case: 2 & File: & MAINDUC1 \\
\hline
\end{tabular}

\section{$3 \times 11$ MAIN DUCT BAKK}

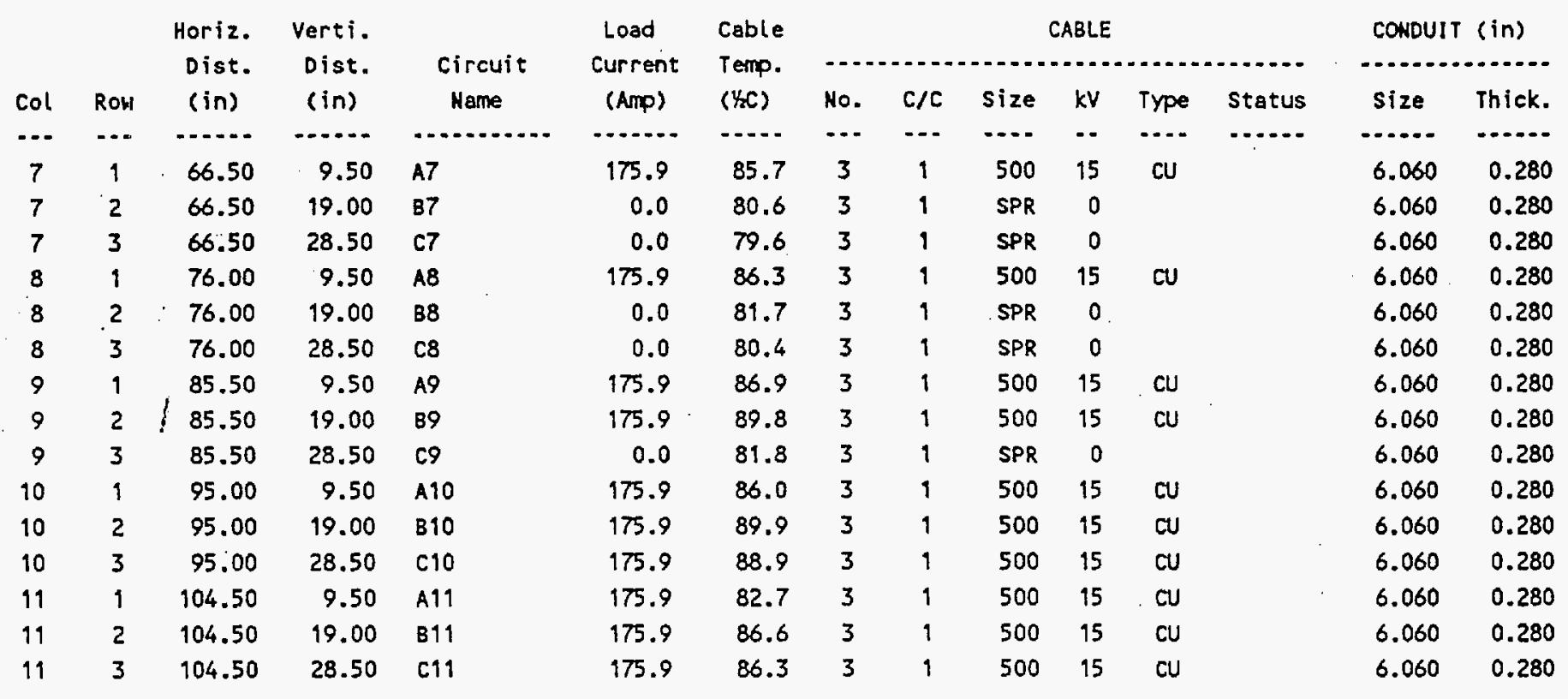




\section{SUPPORT DOCUMENTS (CONTINUED)}

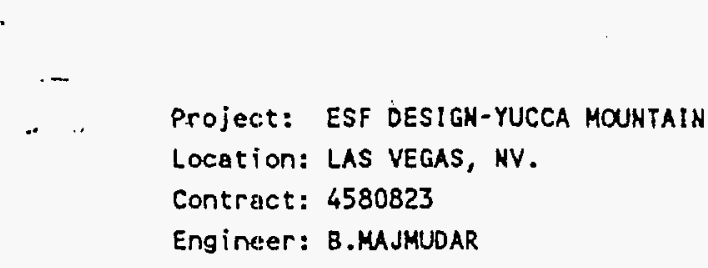

CABLE AMPACITY DERATIHG

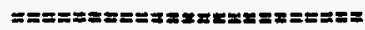

ETAP 6.52

Page: 3

Date: O6-17-1993

SN: $\quad$ B8FD 130132

Engineter: B.MAJMUDAR

Study Case: 2

File: MAINOUC1

\begin{tabular}{|c|c|c|c|c|c|c|c|c|c|c|c|}
\hline & COLUMNS: & 1 & 2 & 3 & 4 & 5 & 6 & 7 & 8 & 9 & 10 \\
\hline & & $\cdots$ & ......... & ;........ & - & - & - & 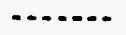 & , & 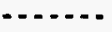 & $\ldots$ \\
\hline & Cable: & 500 & 500 & 500 & 500 & 500 & 500 & 500 & 500 & 500 & 500 \\
\hline ROW 1 & Amp.: & 175.9 & 175.9 & 175.9 & 175.9 & 175.9 & 175.9 & 175.9 & 175.9 & 175.9 & 175.9 \\
\hline & Temp.: & 82.7 & 86.0 & 86.9 & 86.3 & 85.7 & 85.5 & 85.7 & 86.3 & 86.9 & 86.0 \\
\hline & Cable: & 500 & 500 & 500 & SPR & SPR & SPR & SPR & SPR & 500 & 500 \\
\hline ROW 2 & Amp.: & 175.9 & 175.9 & 175.9 & 0.0 & 0.0 & 0.0 & 0.0 & 0.0 & 175.9 & 175.9 \\
\hline & Temp.: & 86.6 & 89.9 & 89.8 & 81.7 & 80.6 & 80.3 & 80.6 & 81.7 & 89.8 & 89.9 \\
\hline & Cable: & 500 & 500 & SPR & SPR & SPR & SPR & SPR & SPR & SPR & 500 \\
\hline ROW 3 & Ampf: & 175.9 & 175.9 & 0.0 & 0.0 & 0.0 & 0.0 & 0.0 & 0.0 & 0.0 & 175.9 \\
\hline & Temp.: & 86.3 & 88.9 & 81.8 & 80.4 & 79.6 & 79.4 & 79.6 & 80.4 & 81.8 & 88.9 \\
\hline
\end{tabular}

\begin{tabular}{crr} 
& COLUMNS: & \multicolumn{1}{c}{11} \\
& & $\ldots \ldots . .$. \\
RON 1 & Cable: & 500 \\
& Amp.: & 175.9 \\
& Temp.: & 82.7 \\
& & \\
& Cable: & 500 \\
ROH $2 \quad$ & Amp.: & 175.9 \\
& Temp.: & 86.6 \\
& & \\
& Cable: & 500 \\
RON 3 & Amp.: & 175.9 \\
& Temp.: & 86.3
\end{tabular}

* Indicates locations where the calculated temperature exceeds the rated conductor temperature es specified in the cable libraries. 


\section{SUPPORT DOCUMENTS (CONTINUED)}

CABLE AMPACITY DERATING

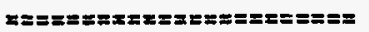

ETAP 6.52
Page: 1

Date: 06-17-1993

SH: $\quad 8 B F D 130132$

File: MAINDUCS

Project:: ESF DESIGH-YUCCA MOUNTAIN

Location: LAS VEGAS, NY.

Study Case: 3

Controct: 4580823
Engineer: B.MAJMUDAR

$3 \times 11$ MAIK DUCT BAKK (750 kemil, $\left./ \mathrm{c}, 9.5^{\mathrm{S}} \mathrm{SPACING,40CAMB.}\right)$

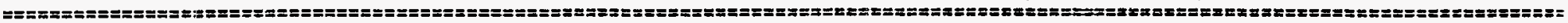

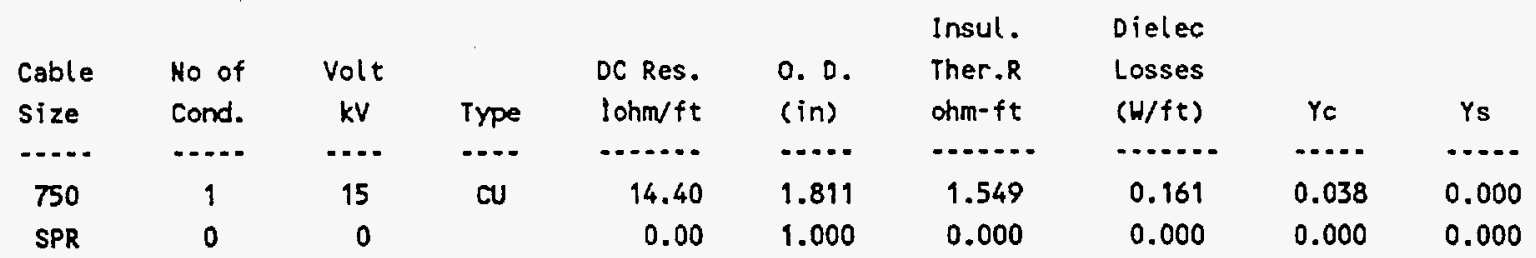

\begin{tabular}{|c|c|c|c|c|c|c|c|c|c|}
\hline & & & & Ref. & & & \multicolumn{2}{|c|}{ RHO } & Ambient \\
\hline Instal - & Conduit & \# of & \# of & Depth & Height & Width & 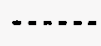 & $\ldots$ & Temp. \\
\hline lation & Type & Rous & Cols & (in) & (in) & (in) & Earth & Fill & $4 x$ \\
\hline 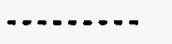 & 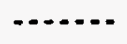 & $\ldots$ & $\cdots$ & 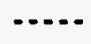 & 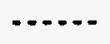 & $\ldots=\ldots$ & $\ldots+$ & $\ldots$. & $\ldots \ldots$ \\
\hline Duct Bank & PVC & 3 & 11 & 24.0 & 38.0 & 114.0 & 120.0 & 85.0 & 40.0 \\
\hline
\end{tabular}

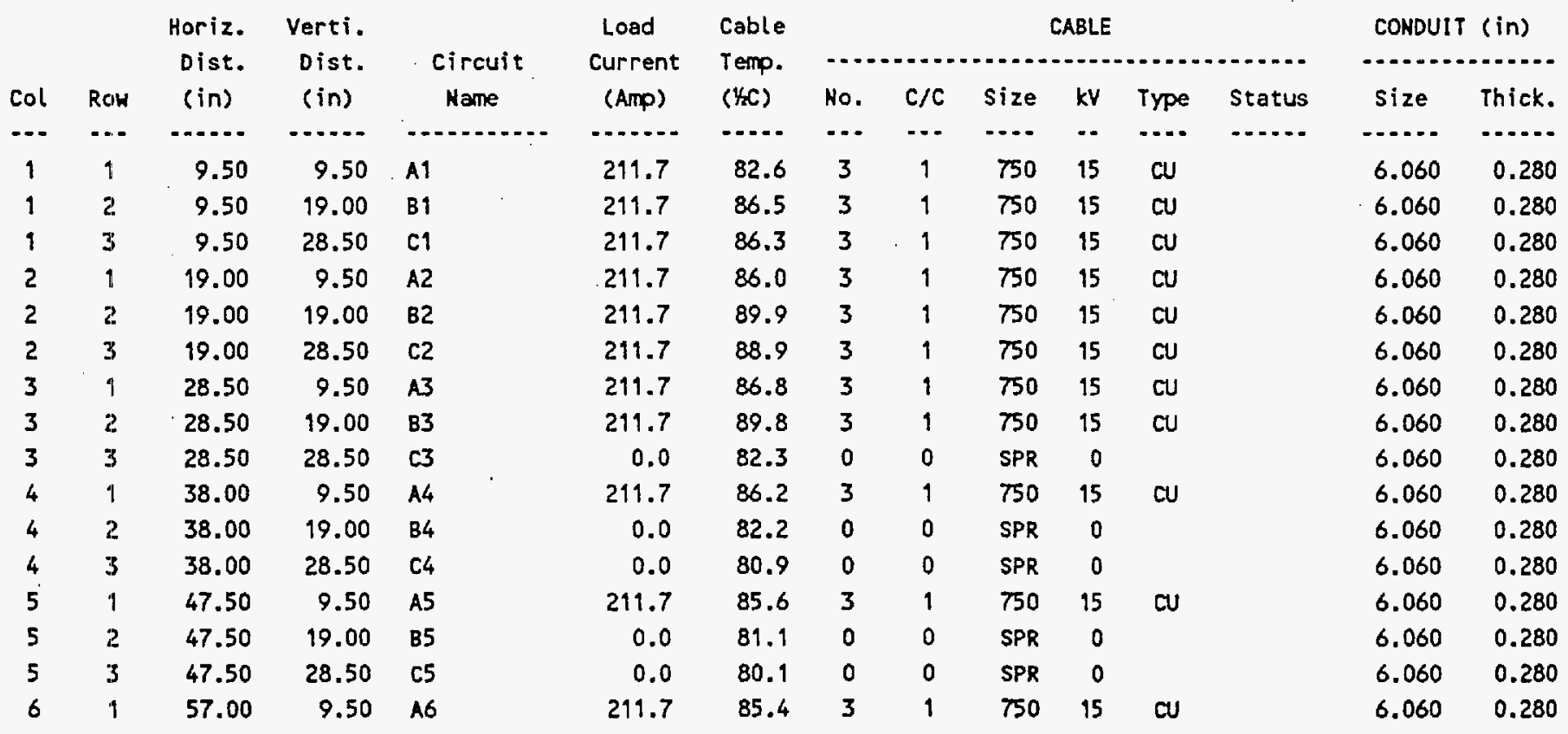




\section{SUPPORT DOCUMENTS (CONTINUED)}

CABLE AMPACITY DERATING

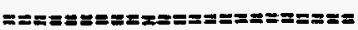

ETAP 6.52

Page: 2

Project: ESF DESIGN-YUCCA MOUNTAIH

Date: 06-17-1993

Location: LAS VEGAS, NV.

SN: $\quad 88 F D 130132$

Contract: 4580823

Engineer: 8.MAJHUDAR

Study Case: 3

File: MAIMOUC5

$3 \times 11$ MAIN DUCT BANK

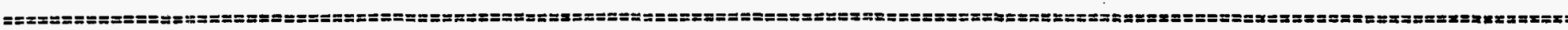

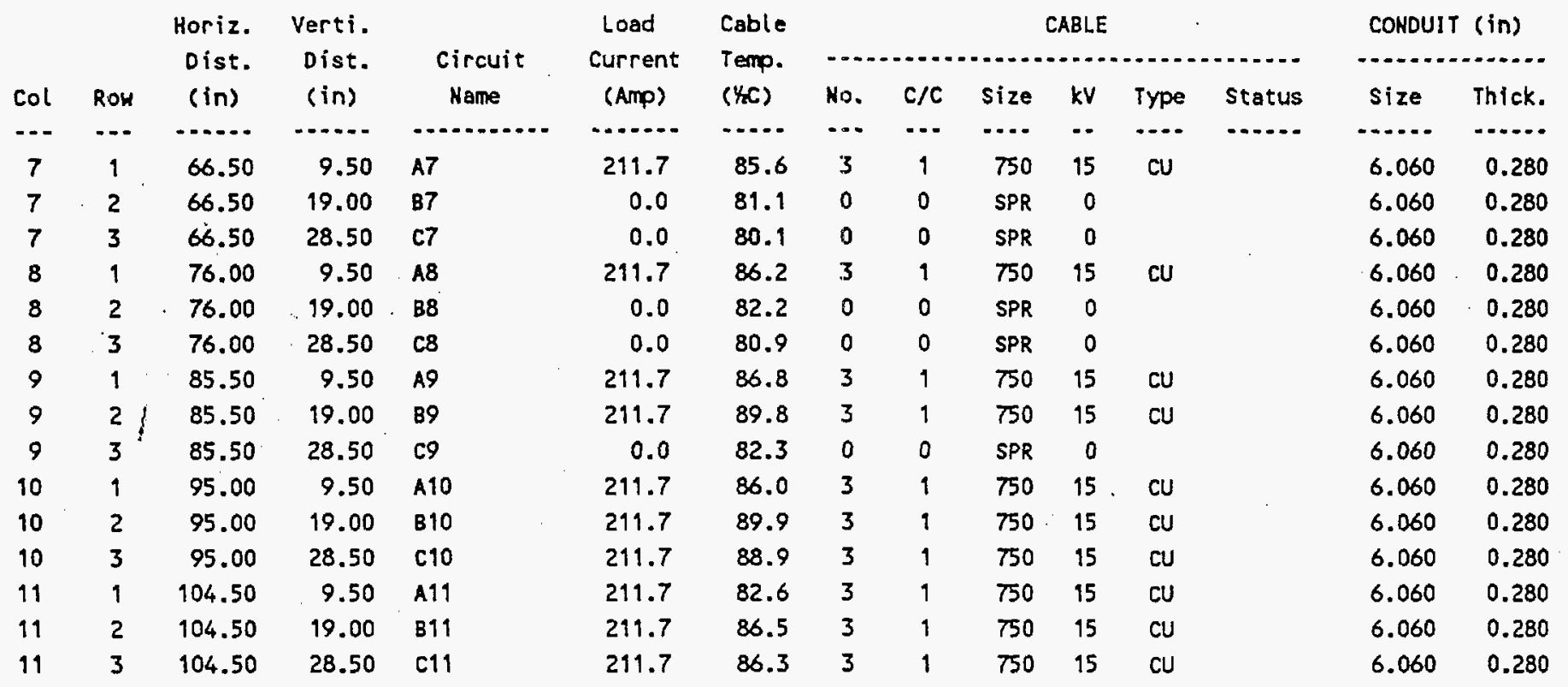




\section{SUPPORT DOCUMENTS (CONTINUED)}

\section{Page: V-11}

CABLE AMPACITY DERATING

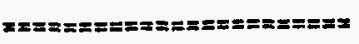

ETAP 6.52

Page: 3

Project: ESF DESIGN-YUCCA MOUNTAIN

Date: 06-17-1993

Location: LAS VEGAS, HV.

SN: $\quad 88 F D 130132$

Contract: 4580823

Engineer: B.MAJMUDAR

Study Case: 3

File: MAINDUC5

\section{$3 \times 11$ MAIH DUCT BAHK}

Filer mainducs

\begin{tabular}{|c|c|c|c|c|c|c|c|c|c|c|c|}
\hline & COLUHNS: & 1 & 2 & 3 & 4 & 5 & 6 & 7 & 8 & 9 & 10 \\
\hline & & $\ldots \ldots$ & - & $\ldots$ & 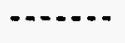 & $\ldots$ & - & 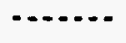 & 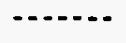 & $\cdots$ & $\ldots$ \\
\hline \multirow{3}{*}{ ROW 1} & Cable: & 750 & 750 & 750 & 750 & 750 & 750 & 750 & 750 & 750 & 750 \\
\hline & Anp.: & 211.7 & 211.7 & 211.7 & 211.7 & 211.7 & 211.7 & 211.7 & 211.7 & 211.7 & 211.7 \\
\hline & Temp.: & 82.6 & 86.0 & 86.8 & 86.2 & 85.6 & 85.4 & 85.6 & 86.2 & 86.8 & 86.0 \\
\hline \multirow{3}{*}{ ROW 2} & Cable: & 750 & 750 & 750 & SPR & SPR & SPR & SPR & SPR & 750 & 750 \\
\hline & Arnp.: & 219.7 & 211.7 & 211.7 & 0.0 & 0.0 & 0.0 & 0.0 & 0.0 & 211.7 & 211.7 \\
\hline & Temp.: & 86.5 & 89.9 & 89.8 & 82.2 & 81.1 & 80.8 & 81.1 & 82.2 & 89.8 & 89.9 \\
\hline \multirow{3}{*}{ ROH 3} & Cablé: & 750 & 750 & SPR & SPR & SPR & SPR & SPR & SPR & $S P R$ & 750 \\
\hline & Almp.: & 211.7 & 211.7 & 0.0 & 0.0 & 0.0 & 0.0 & 0.0 & 0.0 & 0.0 & 211.7 \\
\hline & Temp.: & 86.3 & 88.9 & 82.3 & 80.9 & 80.1 & 79.8 & 80.1 & 80.9 & 82.3 & 88.9 \\
\hline
\end{tabular}

\begin{tabular}{|c|c|c|}
\hline \multicolumn{2}{|c|}{ COLUHNS: } & 11 \\
\hline \multirow{3}{*}{ ROW 1} & Cable: & 750 \\
\hline & Amp.: & 211.7 \\
\hline & Temp.: & 82.6 \\
\hline \multirow{3}{*}{ RON 2} & Cable: & 75o \\
\hline & Amp.: & 211.7 \\
\hline & Temp.: & 86.5 \\
\hline \multirow{3}{*}{ ROW 3} & Cable: & 750 \\
\hline & Amp.: & 211.7 \\
\hline & Temp.: & 86.3 \\
\hline
\end{tabular}

- Indicates locations where the calculated temperature exceeds the rated conductor temperature as specified in the cable libraries. 


\section{SUPPORT DOCUMENTS (CONTINUED)}

\section{Interoffice Correspondence}

Civilian Radioactive Waste Management System

Management \& Operating Contractor

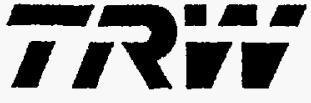

TRW Environmental Safety Systems Inc.
Subject

ESF Subsurface Electrical

Power Requirements

To

Larry Engwall
Date

June 1, 1993

LV.ESSD.JG.6/93-126

cc

P. Pimentel

B. Majmudar

A. Tuma

J. Naaf

W. Reed

E. Eberhartd

W. French

R. Howell
WBS: 1.2 .6

QA: N/A

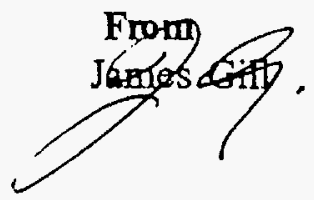

Location/Phone

TES3/4-7999

1

Information requested by the surface design team for properly sizing the feeder cabling to the subsurface facilities has produced the following results:

Two (2) Circuits will be required to power all subsurface equipment and systems excluding the Tunnel Boring Machine (TBM). Each of these circuits will be sized for 25 MVA (1160 A@12.47 $\mathrm{kV}$ ). The two circuits will be connected to provide redundant capacity, total required capacity is estimated at less than 25 MVA.

To provide capacity for $25 \mathrm{MVA}$, nine (9) $3 / \mathrm{C} 500 \mathrm{kcmil}$ cables will be required. This is based on a derated ampacity of $143.3 \mathrm{~A}(1160 / 143.3=8.094)$ to meet NEC requirements 9 cables are required. To provide the redundancy as stated previously two circuits each with nine cables will be provided.

The increased capacity requirement will directly affect the Duct Bank design from the Switchgear Building to the tunnel entrance. The present design has $24-5$ " conduits leaving the Switchgear Building. To accommodate the increased capacity requirement 32 conduits will be planned as follows:

- 9--5"C each with $1-3 / \mathrm{C} 500 \mathrm{kcmil}$ for tunnel $1 \mathrm{~A}$ load

$-9--5^{\prime \prime C}$ each with $1-3 / \mathrm{C} 500 \mathrm{kcmil}$ for tunnel $1 \mathrm{~B}$ load

2--5"C each with 1-3/C $500 \mathrm{kcmil}$ for TBM

2--5"C empty, reserved for future TBM

$1--5^{\prime \prime C}$ with $3 / \mathrm{C}$ (later) for conveyor

$\rightarrow 1--5 " \mathrm{C}$ with $3 / \mathrm{C} 2 / 0$ for surface facility power distribution

8--5"C empty, spare as required by DOE Order $6430.1 \mathrm{~A}$ (25\% spares) 


\section{SUPPORT DOCUMENTS (CONTINUED)}

\section{Interoffice Correspondence}

Civilian Radioactive Waste Management System

Management \& Operating Contractor

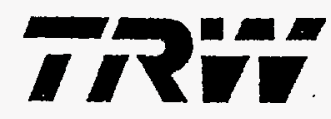

TRW Environmental

Safety Systems Inc.

LV.ESSD.JG.6/93-126

June 1, 1993

Page 2

The increased capacity requirement will necessitate a revision to drawing YMP-025-1-7007-EL109 Rev. 2 and to drawing YMP-025-1-7007-EL128, EL129, \& EL130 all Rev 0. At two locations larger manholes will be required which may require a poured in place design (vs precast). The connections to disconnect switches at the tunnel entrance will require an additional bus bar termination arrangement to accommodate the 9 conductors per phase. This will also enlarge the equipment footprint and cabinet space.

The requirement for the 25 MVA capacity with redundancy should be carefully reviewed with consideration to the following items:

1. The total estimated loading is $\leq 21 \mathrm{MVA}$.

2. Only one $138 \mathrm{kV}$ transmission line feeding the system.

3. Only one $138-12.47 \mathrm{kV}$ transformer at the substation.

4. Only one $12.47 \mathrm{kV}$ line to the main switchgear.

5. The switchgear will operate as a single bus with the tie breaker closed.

The justification for the 25 MVA redundant capacity can only be based on known loading and expected redundant dual primary transmission lines. transformers and incoming service.

When the 25 MVA loading requirement is validated a viable alternative will be to route the cabling above ground in cable tray. A cable tray support stucture is required between the switchgear building and the conveyor structure. The cable tray can be supported by the conveyor structure to the tunnel entrance. This design will increase the cable ampacity by approximatly $60 \%$.

JG:mct 
ATTACHMENT V

1

DI: BABBDA000-01717-0200-00088 Rev. 01

Title: Cable Ampacity Berating

Page: V-14

SUPPORT DOCUMENTS (CONTINUED)

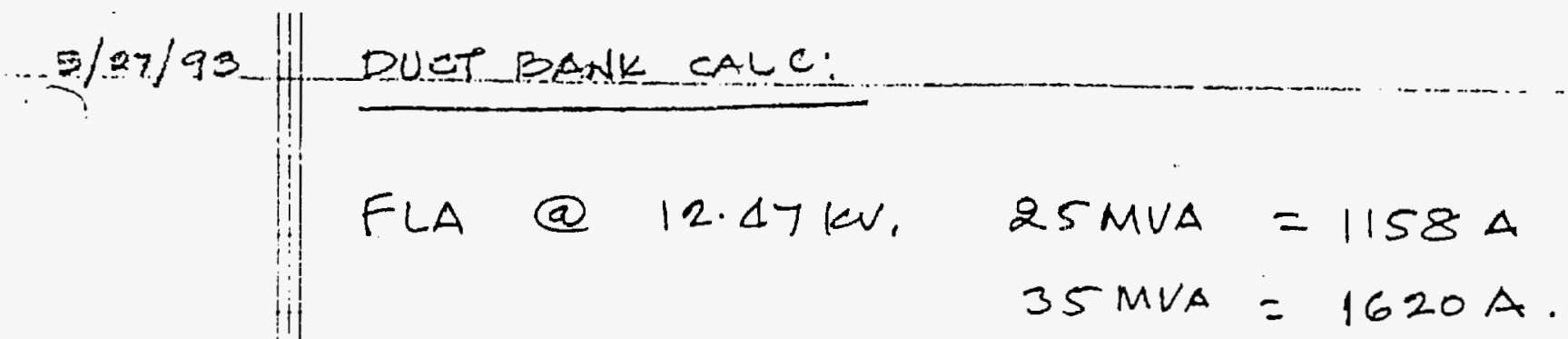

$2 \times 9$ DUCT. BANK

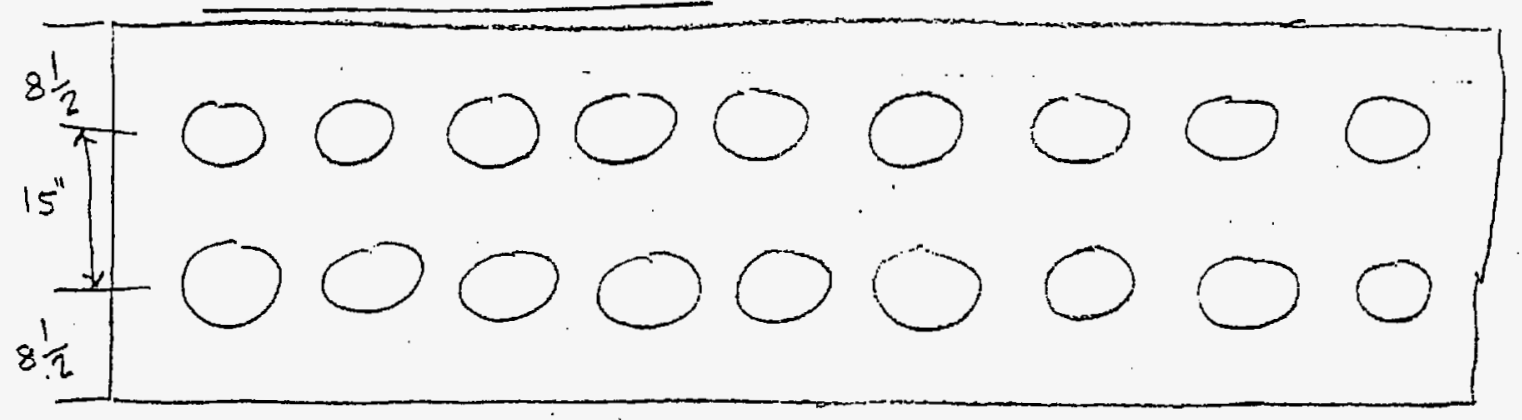

(1)

$$
\text { i CABLE/DUTT. }
$$

(2)

$$
1158 \mathrm{~A}-7-3 / \mathrm{C} 750 \mathrm{MCM}
$$

$1620 \mathrm{~A}-10-3 / \mathrm{C}$ \# $150 \mathrm{MCM}$

$$
(168.6 \mathrm{~A} / \mathrm{cond} \text { ) }
$$

$1158 \mathrm{~A}-$
$(143.3 \mathrm{~A} / \mathrm{cond}$.

$1620 \mathrm{~A}-12-3 / \mathrm{C} \# 500 \mathrm{MCM}$
$(143.3 \mathrm{~A} / \mathrm{Cond})$ 
ATTACHMENT V

DI: BABBDA000-01717-0200-00088 Rev. 01 ।

Title: Cable Ampacity Berating

Page: V-15

SUPPORT DOCUMENTS (CONTINUED)

6 CONDUCTOR PER DUCT.

(3)

$$
\begin{aligned}
& 1158 A- \\
& (104.2 A / \angle A B L E) \\
& 208.4 A / D U C T)
\end{aligned}
$$

$$
\text { 1620A }-16-1 / C-750 \mathrm{MCM} / \mathrm{PHASE}=48-1 / \mathrm{C}-750 \text { MeM }
$$

(4)

$$
\begin{aligned}
& 1158 A \\
& \text { (91.7 A/CABLE } \\
& \text { 183. 4A/DUCT) } \\
& \cdots
\end{aligned}
$$

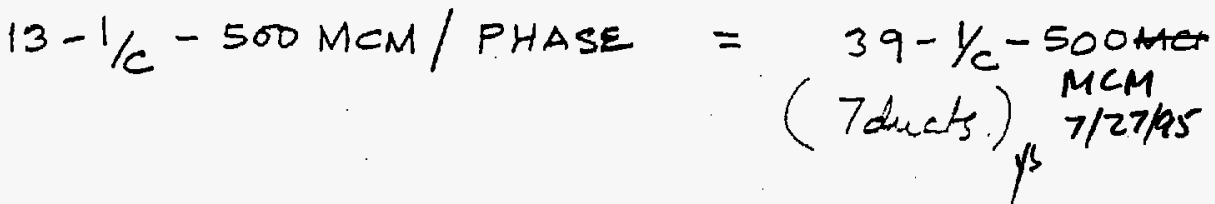

$$
\begin{aligned}
& \text {.. 1620A } \\
& -18-1 / C-500 \mathrm{MCM} / \text { PHASE }=54-1 / C^{-500}
\end{aligned}
$$$$
12-1 / C-750 \mathrm{MCM} / \text { PHASE. }
$$

TOTAL

$$
\begin{aligned}
& =36-1 / \mathrm{c}-750 \mathrm{mcm} \\
& \text { is } 7 / 27 / 95
\end{aligned}
$$

(5)... FrEe AIR RATING:

NE TABLE $310-75$, MB. AIR TEMP. $-40^{\circ} \mathrm{C}\left(104^{\circ} \mathrm{F}\right)$

A. $750 \mathrm{MCM} \longrightarrow 570$ AMPS $\times K$ COR. FACTOR FOR $\left.50^{\circ} \mathrm{C}\right)$ $570 \times 0.93=530$

$$
\therefore 1158 \mathrm{~A} / 530 \mathrm{~A}=3-3 / \mathrm{C}-750 \mathrm{MCM}
$$

B. $\quad 500 \mathrm{MCM} \longrightarrow 470 \times 0.93=437 \mathrm{~A}$

$$
\therefore 1158 \mathrm{~A} / 437 \mathrm{~A}=3-3 / \mathrm{C}-500 \mathrm{MCM} \text {. }
$$


ATTACHMENT V

1

DI: BABBDA000-01717-0200-00088 Rev. 01

Title: Cable Ampacity Berating

Page: V-16

SUPPORT DOCUMENTS (CONTINUED)

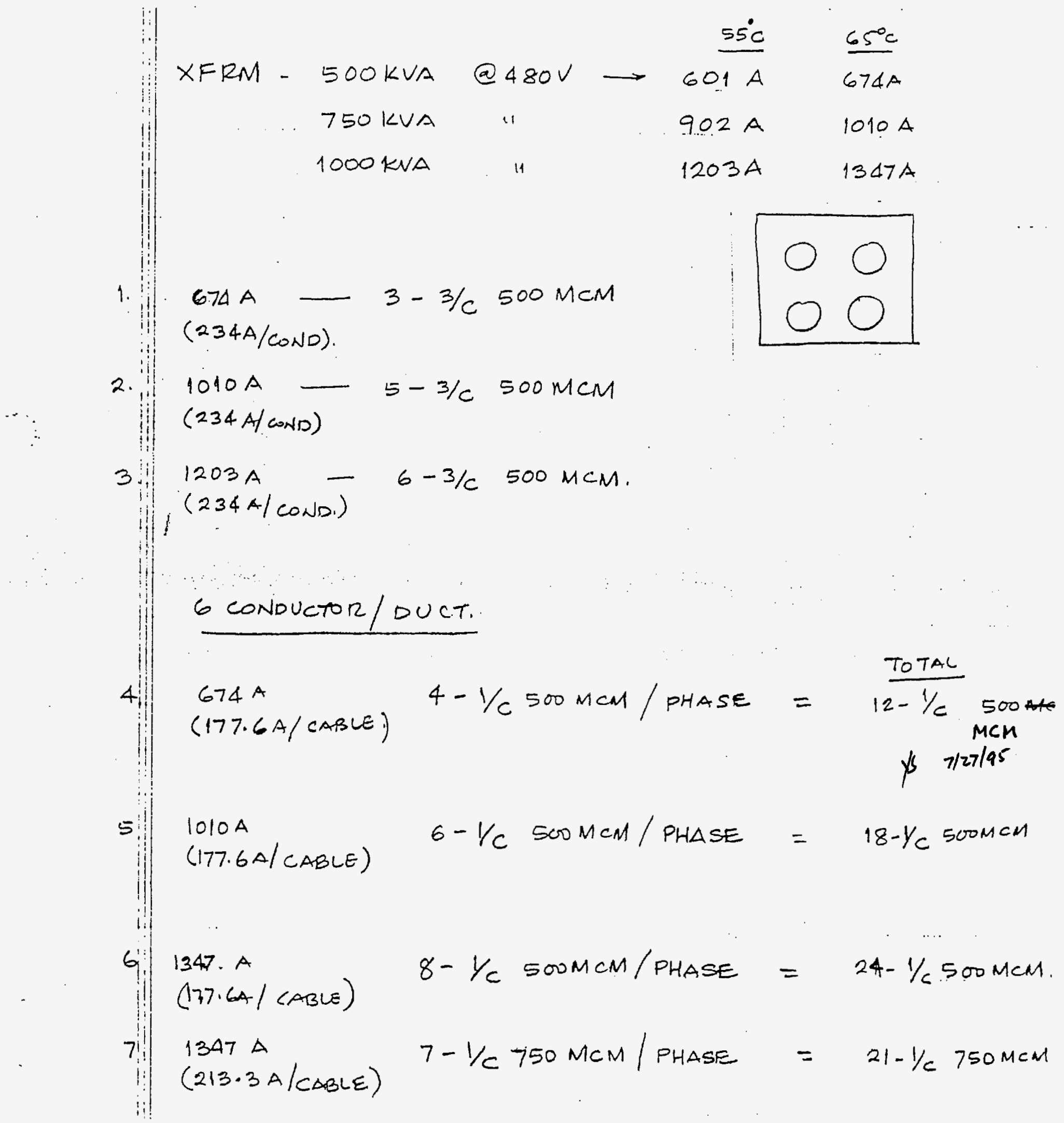




\section{SUPPORT DOCUMENTS (CONTINUED)}

YUCCA MOUNTAIN SITE CHARACTERIZATION PROJECT

Civilian Radioactive Waste Management System

Management \& Operating Contractor

CONTRACT NO. DE-AC01-91RW00134

WUECT: CABLE PERATING - UNDERGROUND DUCT BANK

THE CABLE AMPACITY ADJUSTMENT FAETOR IS COMPOSEO OF FOLLWWNG COMPONENTS

$F=F+F_{t h} \times F_{g}$

WHERE $E_{H}=$ AMBIENT TEMPERATURE AD JUSTMENT EACTOR

$E_{H}=$ SOIL THERMAL RESISTIUITY DIFFERENCE FROM RHO OF 90

$F g=C A B L E$ GROUPING EACTOR. CDEPEAD ON DUCT BANK CONEI GURATION)

USE $\quad F_{t}=0.82$ (WEC 70-174 FOR AMBIENT TEMP OF $46^{\circ}-50^{\circ} \mathrm{C}$ )

$F H=0.90$ (FROM TABLE 3 IEEE PAPER BY SHOKOOH \& KNUTSON)

$F g=U S E$ ATTACHED TABLES FOR CABLE SIZE \& DUCT BANL CONFIGURATION 
ATTACHMENT V

1

DI: BABBDA000-01717-0200-00088 Rev. 01

Title: Cable Ampacity Berating

Page: V-18

SUPPORT DOCUMENTS (CONTINUED)

(i)

$3 / \mathrm{C}$-500 MCM,...5"C

$$
\begin{aligned}
& 0.82 \times 0.69 \times 0.516 \\
& =0.29
\end{aligned}
$$

$$
430 \text { A } \times 0.29=125.54 \text { AMPS }
$$

(2)

$$
1 / c \# 500 \mathrm{McM} \quad \mathrm{s}^{\prime \prime} \mathrm{C} \quad\left[\begin{array}{llll}
0 & 0 & 0 & 0 \\
0 & 0 & 0 & 0
\end{array}\right.
$$

$$
\begin{aligned}
& 0.82 \times 0.69 \times 0.608 \\
& =0.34 \\
& 430 \times 0.34=148 \text { AMPS } .
\end{aligned}
$$

$$
2 \times 9
$$

$$
3 \times 8
$$

$$
\begin{aligned}
& 0.82 \times 0.9 \times 1=0.74 \\
& 0.82 \times 0.9 \times 0.447=0.33
\end{aligned}
$$




\section{SUPPORT DOCUMENTS (CONTINUED) \\ MRS Design}

230 South Tryon Streot

P. O. Box 1004

Charlotte, NC 28201-1004

\section{FACSIMILE COVER SHEET \\ FAX No. (704) 382-3105}

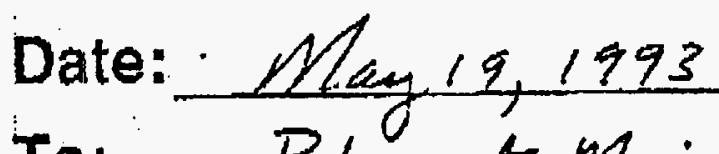

To: Bharat Majmudar

Company: $M \div 0$

FAX No.: $\frac{(702) 794-1843}{-2}$

From:

Bob Howee

Telephone No.: (704) $38 z-3086$

\section{Message:}

Dappen documentation fo $757 \mathrm{man}$ cable application on 5 tombloy genentors

This Transmittal Contains _... 7 Pages Including Cover Sheet 


\section{SUPPORT DOCUMENTS (CONTINUED)}

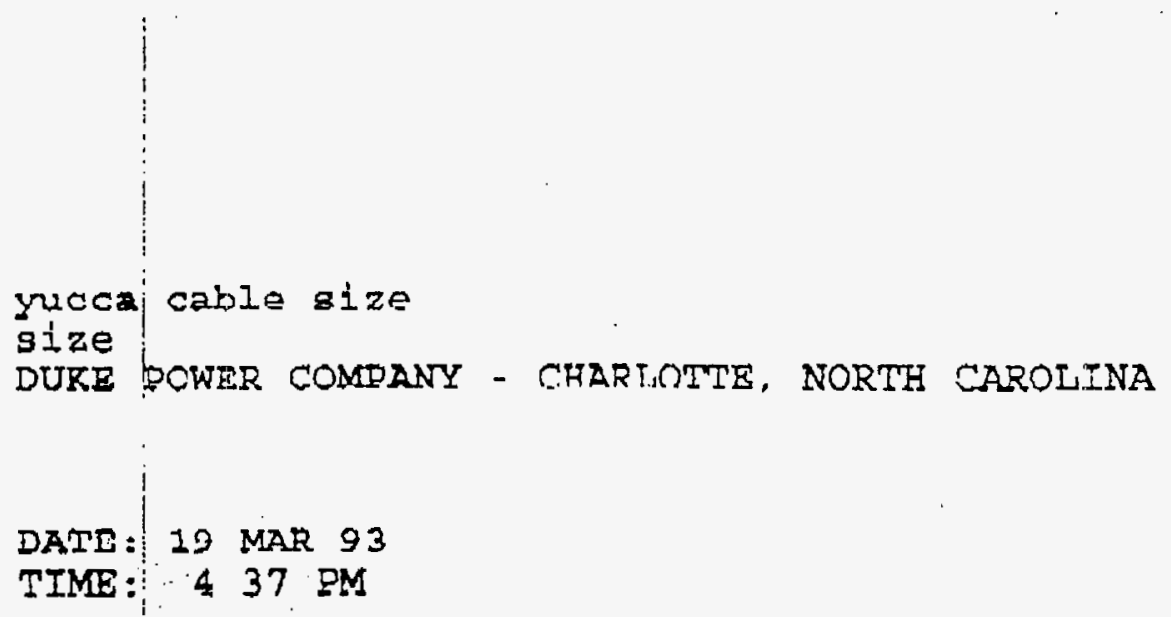




\section{SUPPORT DOCUMENTS (CONTINUED)}

\section{LATE: 19 MAR 93 TIMB: 437 PM}

Yicca cable size

size

DUKE POHER COMPANY - CHARLOTTE, NORTH CAROLINA

i.j.

FEBDER AND TRANSFORMER STUDY CRITERIA

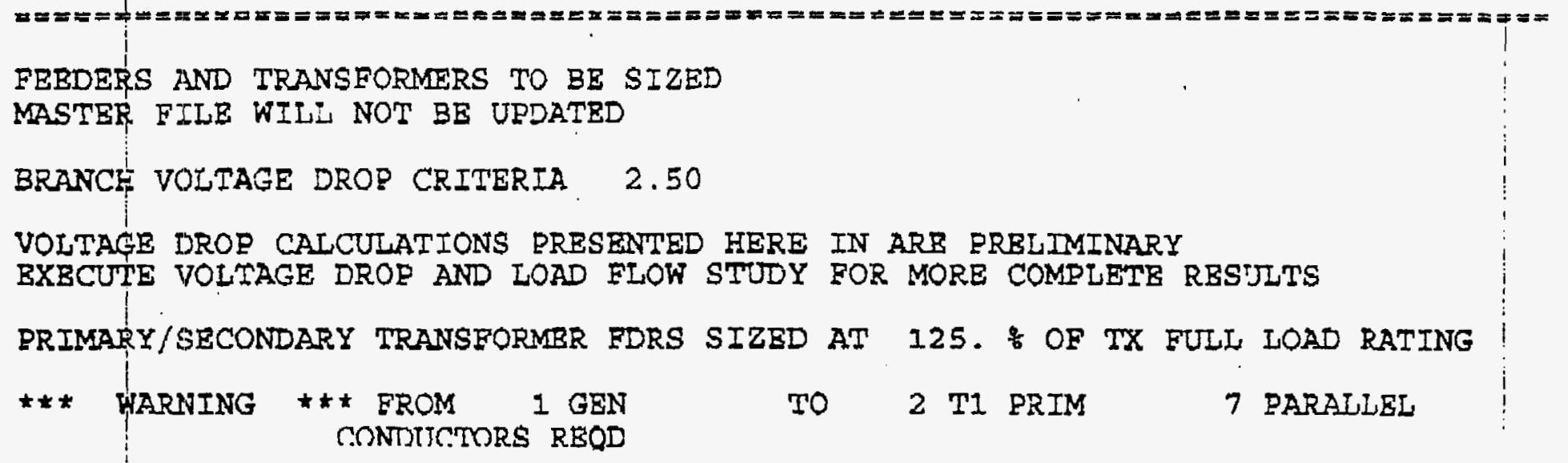

FEEDERS AND TRANSEORMERS TO BE SIZED

ARR FILE KILL NOT BE UPDATED

VOITACR DROP CALCULATIONS PRESEMTED

PRIMARY/SECONDARY TRANSFORMBR FDRS SIZED AT 125.

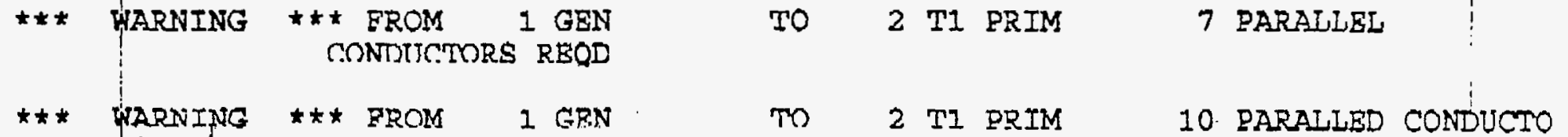

** HOTICE ** FEEDER SIZED TO 125. PERCENT OF TRANSPORMER SIZE

BRANCH FROM 1 GEN TD 2 T1 PRTM

TR KVA: 2300.0 TR ELA: 2766.5

MINIMUM FEEDEP AMPACITY: 3458.1 
SUPPORT DOCUMENTS (CONTINUED)

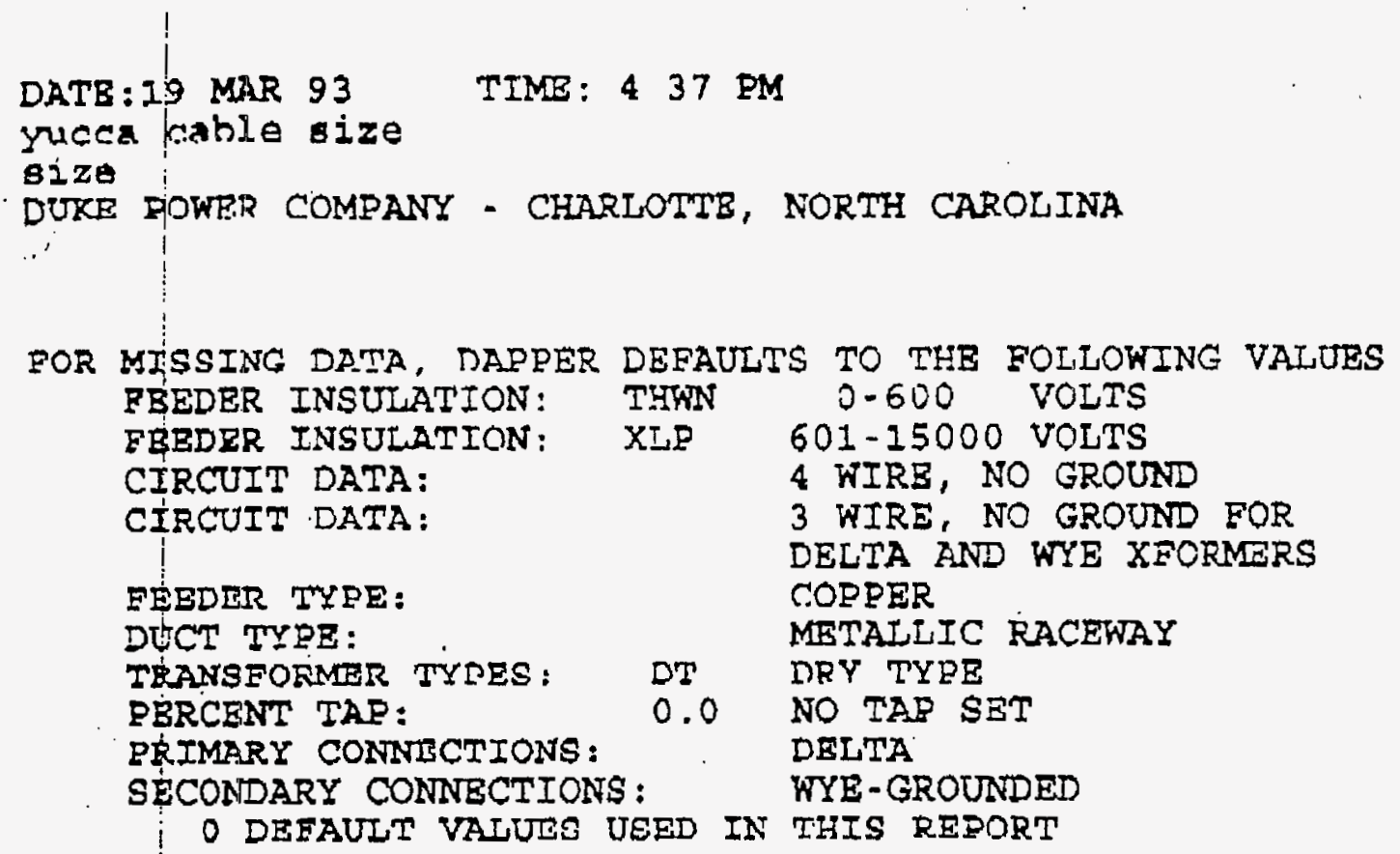


SUPPORT DOCUMENTS (CONTINUED)

DATB:19 MAR 93 TIMB: 437 FM

rucca cable aize

size

UKE POWER COMDANY - CHARLOTTE, NORTH CAROLINA

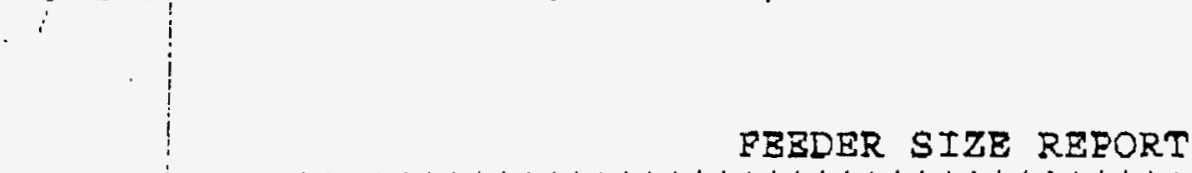

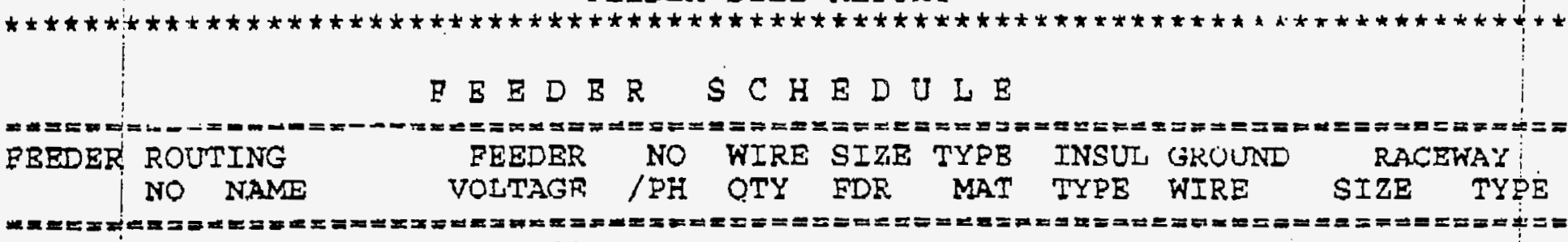
FROM 1 GEN 480 . 
ATTACHMENT V

1

DI: BABBDA000-01717-0200-00088 Rev. 01

Title: Cable Ampacity Berating

Page: V-24

SUPPORT DOCUMENTS (CONTINUED)

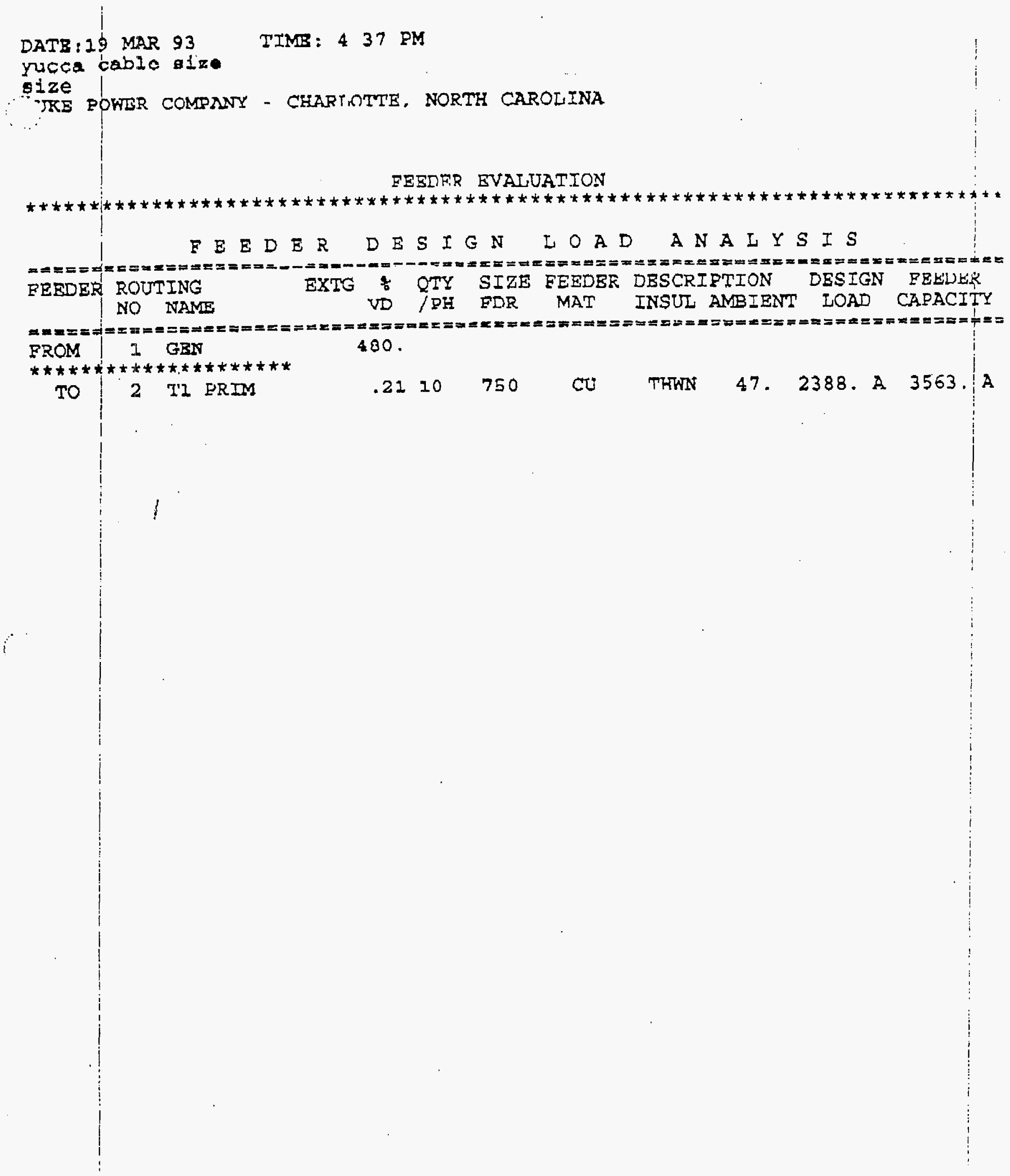




\section{SUPPORT DOCUMENTS (CONTINUED)}

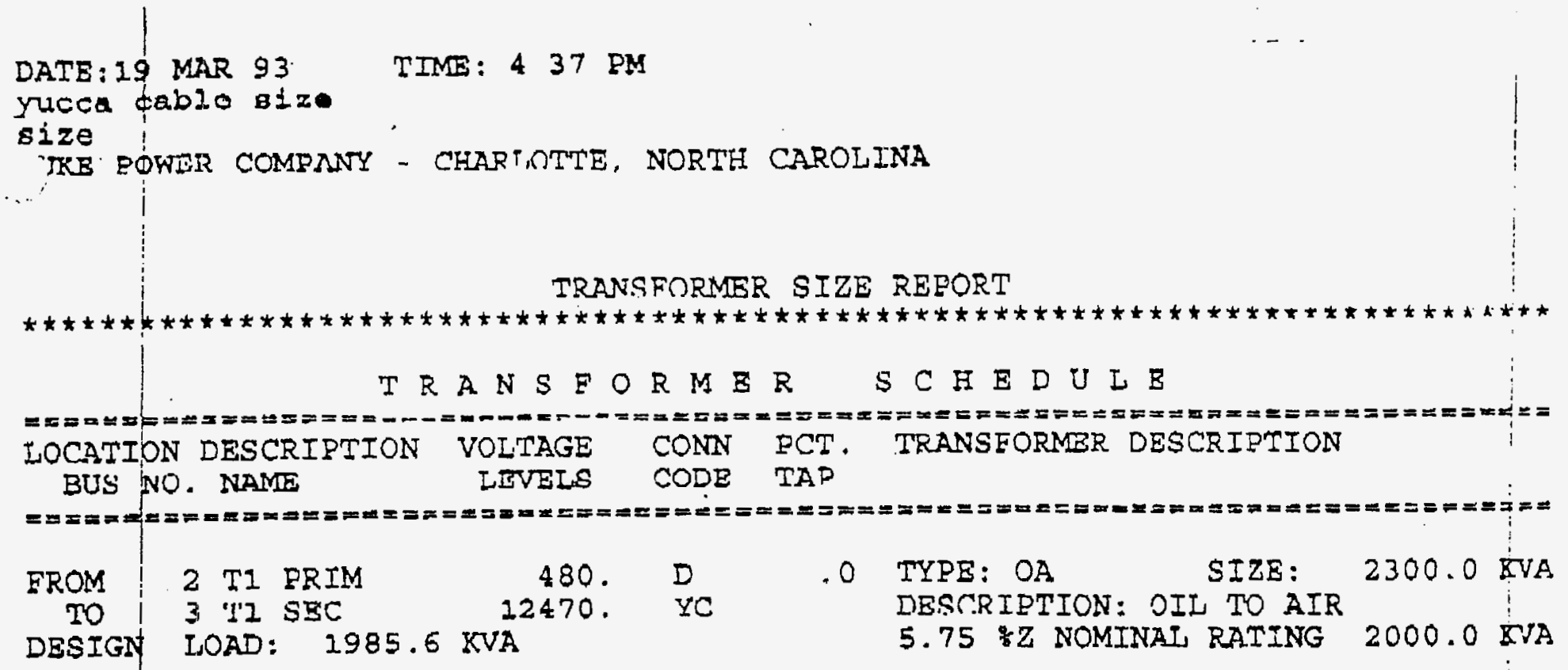


SUPPORT DOCUMENTS (CONTINUED)

MRS Design

230 South Tryon Street

P. O. Box 1004

Chartotte, NC 28201-1004

\section{FACSIMILE COVER SHEET \\ FAX No. (704) 382-3105}

Date: S118193

To: RS Howill

Company: DEdS

FAX No.:

From:

Rloningt:

Telephone No.: $\quad-704-382-3086$

Message:

This Transmittal Contains 11 Pages Including Cover Sheet 


\section{SUPPORT DOCUMENTS (CONTINUED)}

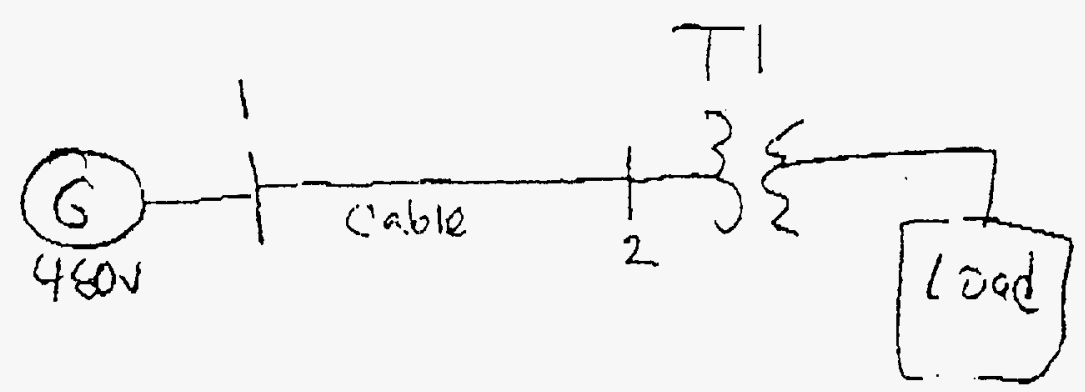

yucca demand load analysts

size

DURE POWER CUMPANY - CHARLUTTE, NORTH CAROLINA

DATE: 18 MAR 93

TIME : $945 \mathrm{AM}$

ALL INRORMATION DRESEITTED IS FOR REVIEW, APDROVAL, INTERPRETATION

ID AFPLICATION BY A, REGISTRRED ENGINEER ONLY

IADPE? (DEMAND LONI ANALYSIS MINI/MICRO VERSICN 3.5 LEVEL 2.1)

COPYRIGHT SKM SYSTZMS ANAIYSIS, INC. 1983 


\section{SUPPORT DOCUMENTS (CONTINUED)}

DATB: 2 के MAR 93 TIMB: 945 AM

rucca demard load analysia

pize i

is POWER COMRANY - CHARIOTTE, NORTH CAROTINA

LOAD SUMMARY

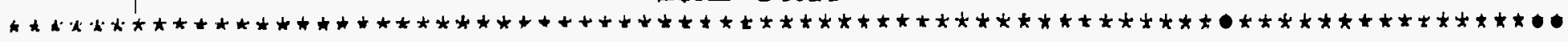

LOAD SCHEDLLE FCR 1 GSN

SOURCE OR PWR $\$ * \star \star$ SOURCE BUS

Page: V-28

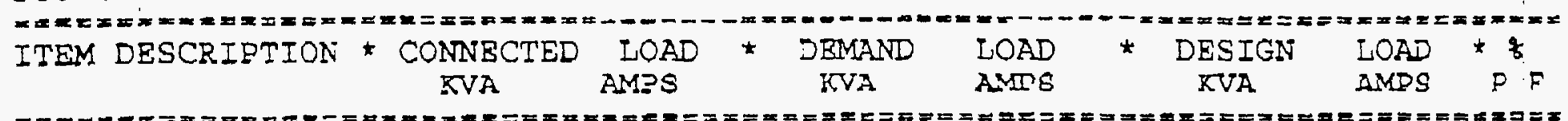

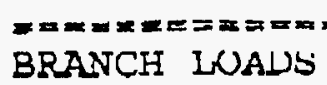

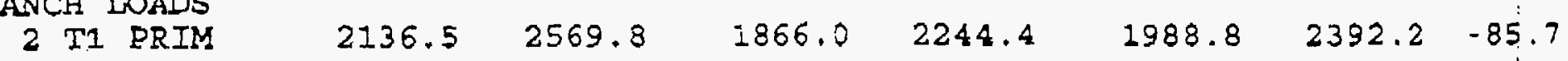

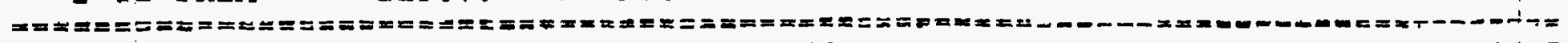

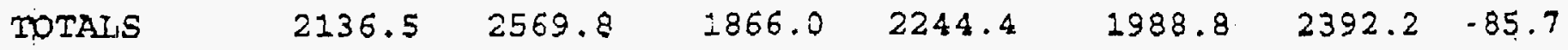

$\begin{array}{ll}\text { LOAD SCEEDULE FOR } & 2 \mathrm{~T} \text { T FRLM } \\ \text { SOURCE OF FWR } & 1 \text { GEN }\end{array}$

480. VOLTS LINB TO I.INE

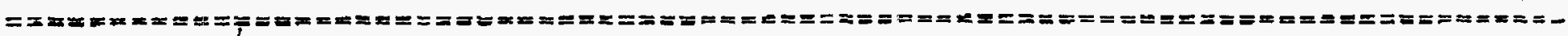
ITEM DESCRIETION * CONN3CTED LOAD * DEMAND IOAD * DESIGN LOAD *

KVA AMPS KVA AMES SVA AMPS $F ;$

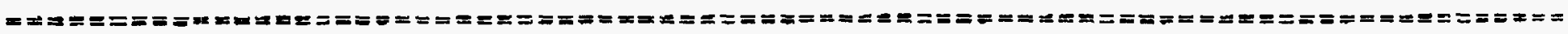
BRANCH LOADS

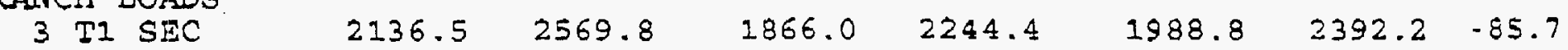

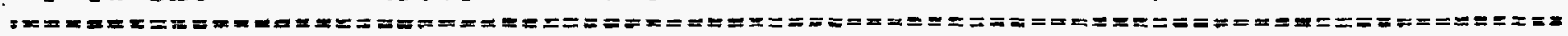
$\begin{array}{llllllll}\text { TOTAJS } & 2136.5 & 2569.8 & 1866.0 & 2244.4 & 1988.8 & 2392.2 & -85.7\end{array}$

LOAD SCHEDULE FOR 3 TI SEC

SOURCE OE PWR 2 TI PKIM

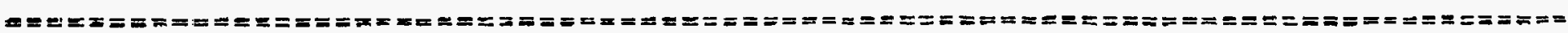
ITEM DESCRIPTION * GONNACTFD LOAD * DIMAND LOAD * DESIGN LOAD * KVA ANPS KVA AMPS KVA AMPS $E E$

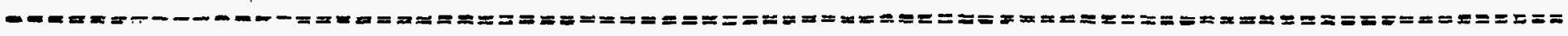
END USE LOADS

CENERAT LOADS

LIGHTING

RBCEPTACLES

MOTOR LOADS

LARGEST MOTOR

500.0
300.0

.156 .0

1000.0

$-200.0$

$== \pm== \pm== \pm=$ TOTALS
2136.3
23.1
13.9
7.2
46.3
9.3
$30 n .0$
83.0
1000.0
200.0

$98.9 \quad 1866.0$

13.9

13.9

3. 8

46.3

9,3

\section{0}

375.0

83.0

1000.0

2.50 .0

$\begin{array}{cc}=z==\pi & ======== \\ 86.4 & 198.8\end{array}$
$13.9-90.0$

$17.4 \quad .95 .0$

$3.8 \quad-95.0$

$46.3-80.0$

$11.6 \quad-80.0$

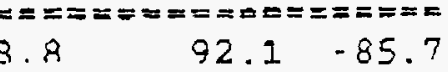




\title{
SUPPORT DOCUMENTS (CONTINUED)
}

\author{
DATE:18 MAR 93 TIME: 945 AM \\ yecca demard load analyats \\ size 1 \\ TE POWER COMPANY - GHARLOTTE, NORTH GAROT.TNA
}

SOURCB IOAD SUMMARY
SUt...

IOAD BUYS 1 GEN

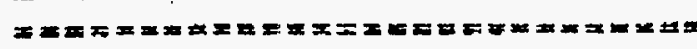

LOAD DESCRIPTION UNITS

TYPE

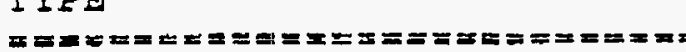

GENERAI LOADS

(1)

LIGHTING

RECEPTACLES

MOTOR LOADS

i

IAREEST MOTOR

\section{KN}

KVAR

KVA

XT

KVAR

KVA

$\overline{X W}$

KVAR

KVA

KW

KVAR

RVA

KW

KVAR

TVA

\begin{abstract}
CONNECTISD
\end{abstract}
LOAD

480. VOLTS LINE TO TITNR.

450.0

$-217.9$

500.0

285.0

$-93.7$

300.0

148.2

$-48.7$

156.0

800.0

.600 .0

1000.0

160.0

$-120.0$

200.0 $=x=x=x$
DEMAND LOAD
DESIGN

LOAD

$=-m-\infty$

270.0

$-130.0$

300.0

356.3

.117 .1

375.0

78.8

$\begin{array}{rr}23.9 & .25 .9 \\ 83.0 & 83.0\end{array}$

$\begin{array}{rr}-23.9 & .25 .9 \\ 83.0 & 83.0\end{array}$

800.0

$-600.0$

1000.0

200.0

$-150.0$

250.0

$\begin{array}{rr}-130.8 & -130.8 \\ 300.0 & 300.0\end{array}$

90.0 LAGGING DOWER FACTOR. \&

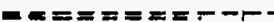

95.0 LAGGING

$-600.0$

1000.0

160.0
120.0
200.0

1593.8

$-970.4$

1866.0

85.4

LAGGING LAGGING LAGGING

1705.1

.1023 .8

1988.8

85.7 


\section{SUPPORT DOCUMENTS (CONTINUED)}

DATB: 18 MAR 93 TIME: 945 AM

yucca demand load analysis

size

DUKB PCWER COMPANY - CHARLOTTE, NORTH CAROLINA

\section{LOAD DEMAND TABLE}

\begin{tabular}{|c|c|c|c|c|c|c|c|c|c|}
\hline IOAD DESCRIPTION & $\begin{array}{l}\text { LOAD } \\
\text { TYPE }\end{array}$ & $\begin{array}{r}\text { FIRST } \\
\text { RVA }\end{array}$ & $\begin{array}{c}\text { DEMAND } \\
*\end{array}$ & $\begin{array}{r}\text { SECOND } \\
\text { KVA }\end{array}$ & $\underset{t}{\text { DEMAND }}$ & $\begin{array}{c}\text { THIRD } \\
\text { KVA }\end{array}$ & $\underset{t}{\text { DEMAND }}$ & $\begin{array}{l}t \\
P E\end{array}$ & $\begin{array}{l}\text { DESIGN } \\
\text { FACh }\end{array}$ \\
\hline & & & & & & & & $=\geq x$ & \\
\hline GENBRAI LOADS & $\mathbb{R}$ & 100 . & 100. & ALL & 50 & $A \amalg L$ & 50. & -90.0 & 1.00 \\
\hline LIGHTTNG & $\mathrm{K}$ & AII & 200. & ALL & 100. & $\lambda I L$ & 100 & -95.0 & 1.25 \\
\hline RECBPTACLES & 2 & 10. & 100. & ALL & 50 & ALL & 50 . & $-95 . C$ & 1.00 \\
\hline OPFICA EQUIPMENT & $z$ & AIL & 100. & MLL & 100 & ALL & 100 & -05.0 & 1.00 \\
\hline HEATING & $\bar{z}$ & $A L L$ & 100. & ALL & 100. & ALI & 100 & 100.0 & 1.25 \\
\hline STANDRY LOAOS & $\bar{R}$ & $\overline{A I L}$ & 100. & ALL & 100 . & ALL & 100 . & .85 .0 & 1.25 \\
\hline CABACITOR BANK & $\mathbf{z}$ & ALL & 100 . & ALL & 100. & ALL & 100. & .0 & 1.35 \\
\hline SYNC, MOTOR & $\mathrm{K}$ & ALL & 100 & ALL & 100 & AIL & 100. & 100.0 & 1.25 \\
\hline MOTOR IUADS & $\mathrm{K}$ & ALL & 100. & ALL & 100 & ALL & 100. & -80.0 & 1.00 \\
\hline INRGEST MOTOR & $\mathrm{x}$ & AUI & 100. & ALI & 100. & ALI & 100 & -80.0 & 1.25 \\
\hline CRANE & $\mathrm{K}$ & ALL & 20 & ALL & 20 & ALL & 20 . & .80 .0 & 1.00 \\
\hline MOTOR & $\pi$ & ALL & 50. & ALI & 50. & AIL & 50. & -80.0 & 1.00 \\
\hline MOTOR & $\mathrm{K}$ & AUL & 70 & ALL & 70 & AIL & 70 & -80.0 & 1.00 \\
\hline SPARE & $K$ & ALI & 100. & $A I L$ & 100. & ALL & 100 & 100.0 & 1.00 \\
\hline SPARE & $\mathrm{x}$ & AII & 100. & ALL & 100. & AIL & 100 & 100.0 & \\
\hline SPARB & $\boldsymbol{x}$ & AIL & 100. & LLL & 200. & ALL & 100. & 100.0 & \\
\hline SPARE & $\mathrm{K}$ & ALI & 100. & ALL & 100. & ALL & 100 & 100.0 & \\
\hline SPARE & $\mathrm{K}$ & Lلـا & 200. & $A L L$ & 100. & ALL & 100. & 100.0 & \\
\hline SPARE & $\mathbf{K}$ & ALL & 100. & ALI & 100 & ALI & 100. & 100.0 & 1. \\
\hline SPARE & $\mathrm{k}$ & ALL & 100. & ALL & 100 . & ALL & 100 & 100.0 & 1.00 \\
\hline
\end{tabular}

NOTES: LOAD TYPE 10 PROVIDES TRANSFER PUNCTION TO LOAD TYPE 9 DEMAND AND DESIGN FACTCRS APPLIED AT EACH LOAD BUS AND AIL LOAD TOTALS ARE EOWER FACTOR CORRECTBD 


\section{SUPPORT DOCUMENTS (CONTINUED)}

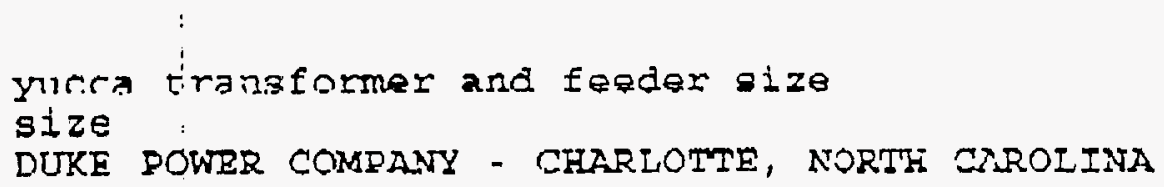




\section{SUPPORT DOCUMENTS (CONTINUED)}

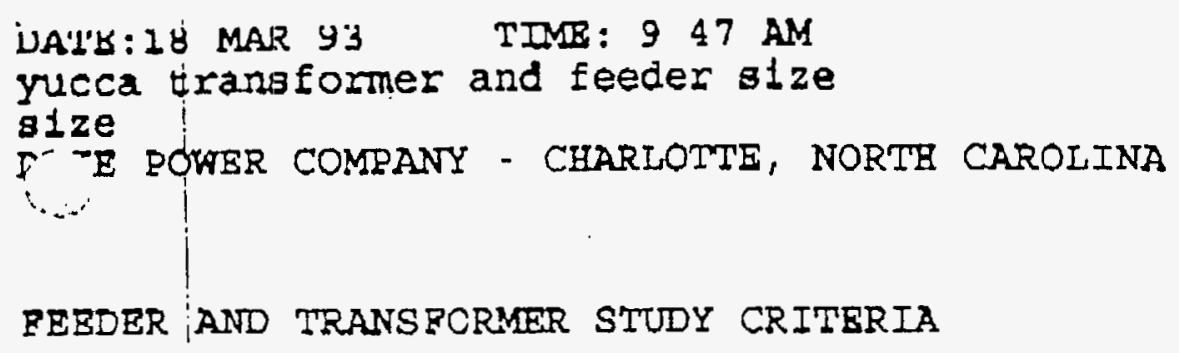




\section{SUPPORT DOCUMENTS (CONTINUED)}

DATB:I8 MAR 93 'LIMS: 947 AM

yucca transformer and feeder bize

sıze

ITKB PQWER COMPANY - CHARLOTTZ, NORTH CAROLINA

$\because \because "$

FOR MISSING DATA, DAPPER DEFAULTS TO THE FOLLOWING VALUES

FEEDRR INSULATION: THWN 0.600 VOLTS

FEEDER INSULATION: XLP $601-15000$ VOLTS

CIRCUIT DATA:

4 WIRE, NO GROUND

CIRCUIT DATA:

3 WIRE, NO GRCUND FOR

FEEDER TYPE:

DUCCT TYPE:

DELTA AND WYE XFORMERS

COPPER

TRANSFORMER TYPES: DT DRY TYPE

PERCENT TAP: $\quad 0.0$ NO TAP SET

RRIMARY CONNTCTIONS: DELTA

SECOONDARY CONNBCTIONS : WYE-GROUNDED

OO DEFAULT VALUES USED IN THIS REPORT 


\section{SUPPORT DOCUMENTS (CONTINUED)}

DATB: 18 MAR 93 TIME: 947 AM

yucca transformer and feeder size

glze

F.- $\mathrm{E}$ POWER COMPAVY - CHARLOTTE, NORTH CAROLINA

$\checkmark j$

\section{FEBDER SIZE REPORT}

FEEDER SCHEDULE

FEEDER TROUTING

NO NAMB

FEEDER

VOT,TARR

No

WIRE SIZE TYPE

INSUL GROJND

RACEWAY

Page: V-34

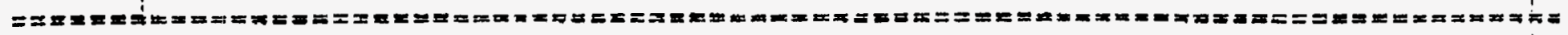
FROM 1 GEN

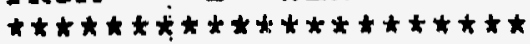

TO 2 TI DRIM

(13) 3 500 OU TTHWN

$N$

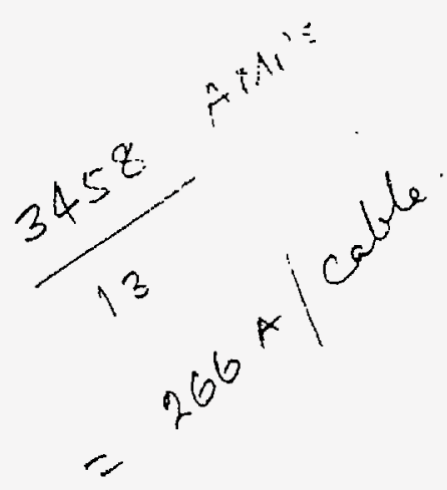


ATTACHMENT V

DI: BABBDA000-01717-0200-00088 Rev. 01

Title: Cable Ampacity Berating

Page: V-35

SUPPORT DOCUMENTS (CONTINUED)

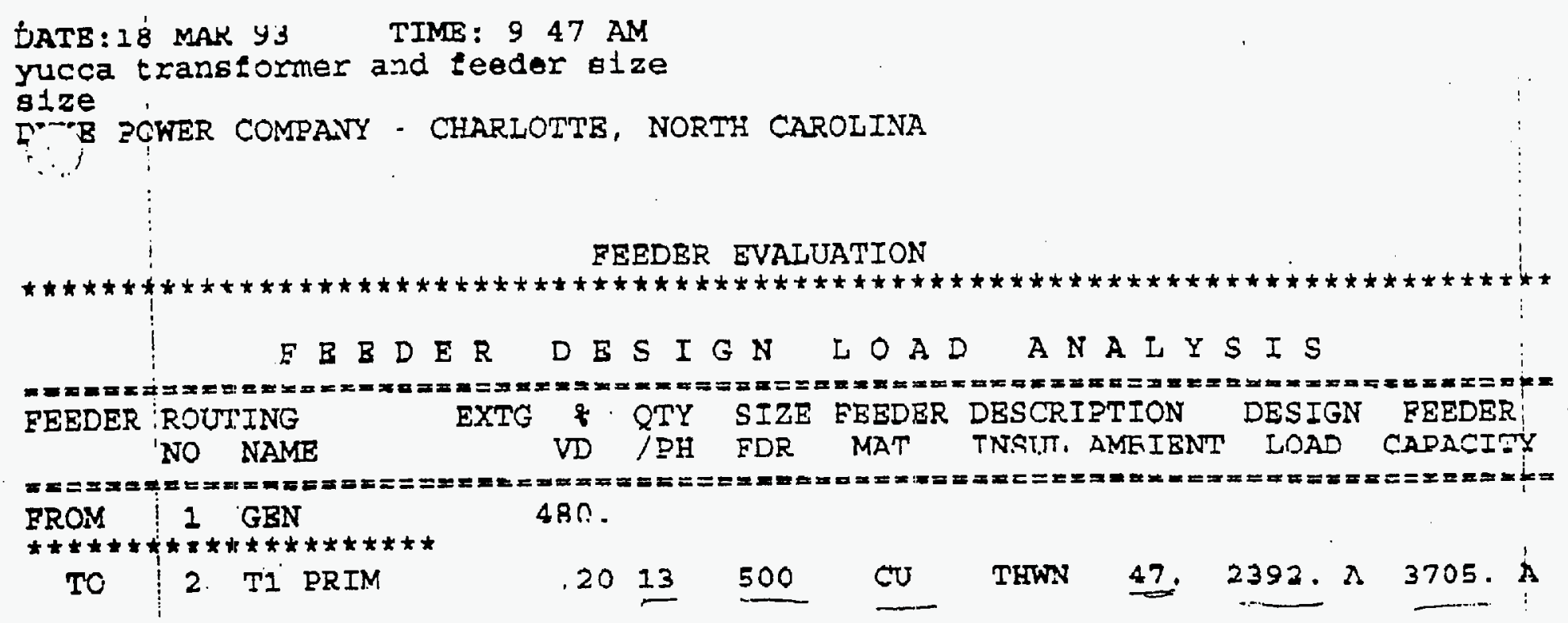




\section{SUPPORT DOCUMENTS (CONTINUED)}

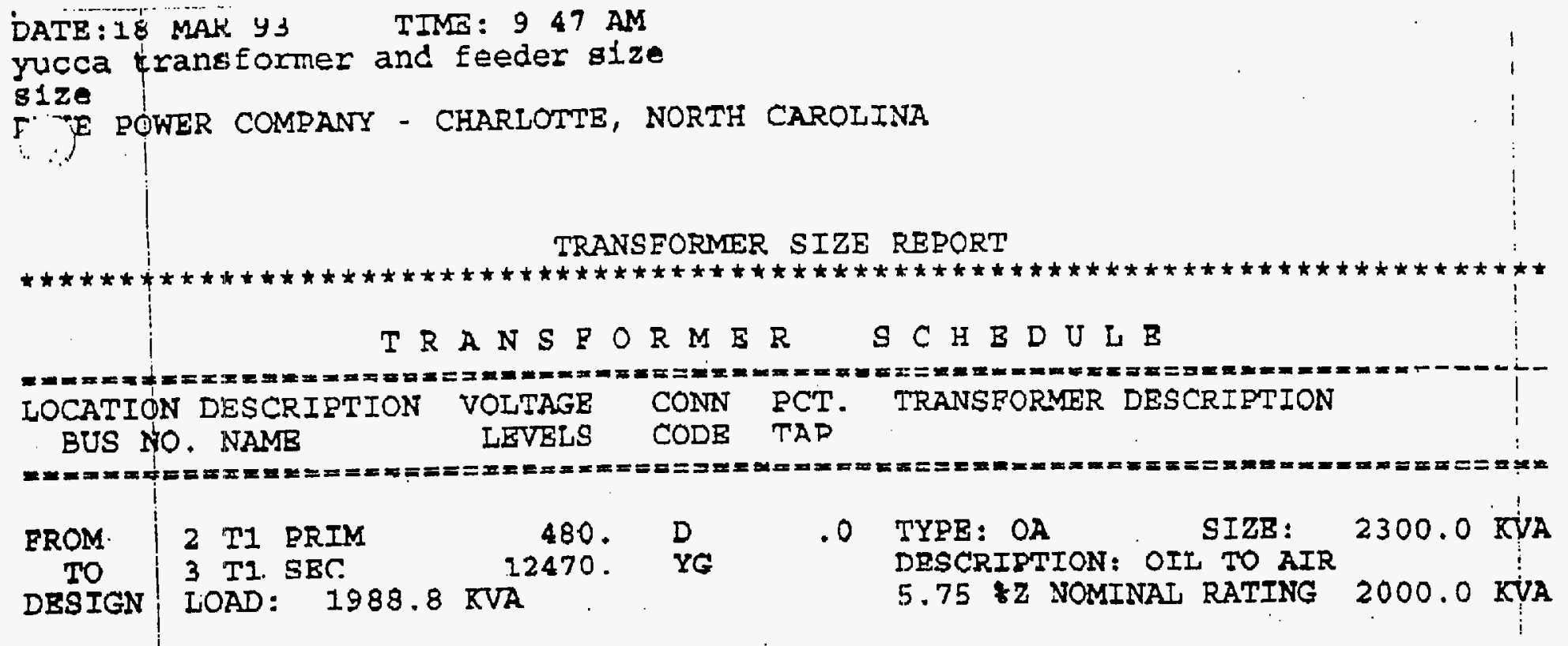




\section{SUPPORT DOCUMENTS (CONTINUED)}

ACTIUITY REPDRT 〈SEND〉

\begin{tabular}{|c|c|c|c|c|c|c|c|}
\hline NO. & REMOTE STATION & MODE & START & DURATION & PAGES & RESULT & NOTE \\
\hline 006 & 7149755271 & STD & $17.11: 43$ & $11: 40$ & 24 & OK & SEND \\
\hline 007 & 7027941843 & STD & $17,12: 00$ & $01 \cdot 35$ & 04 & OK & SEND \\
\hline 008 & $453 ? 8$ & STD & $17.13: 49$ & $02 \cdot 14$ & 04 & OK & SEND \\
\hline 019 & 3026424560 & STD & $17,14: 56$ & $00 \cdot 58$ & 02 & OK. & SEND \\
\hline 010 & $714 \quad 975 \quad 5949$ & STD & $17,15: 16$ & $03 \cdot 11$ & 05 & $O K$ & SEND \\
\hline 011 & $8909382236 ?$ & STD & $17.15: 58$ & $00^{\prime} 00$ & 00 & $* 0.0 .0$ & SEND \\
\hline 012 & $714 \quad 382 \quad 2868$ & STD & $17,15: 59$ & $03 \cdot 1 ?$ & 05 & * OK & SEND \\
\hline 001 & $702 ? 341843$ & STD & $18,08: 10$ & 02.46 & 07 & $D K$ & SENE \\
\hline 002 & 7149755816 & STD & $18,08: 24$ & $01 \cdot 15$ & 02 & OK & SEND \\
\hline
\end{tabular}

ACTIWITY REPDRT SRECEIVE)

\begin{tabular}{|c|c|c|c|c|c|c|c|}
\hline NO. & REMOTE STATION & MODE & START & DUURRTION & PAGES & RESULT & NOTE \\
\hline 008 & $702 \quad 246 \quad 7300$ & STD & $17,11: 38$ & $02 ' 14$ & 02 & OK & \\
\hline $\cos 3$ & $6:$ & STD & $17,12: 38$ & 02.31 & 02 & $0 k$ & \\
\hline 010 & $702 \quad 295=515$ & $\dot{S T T}$ & $17,13: 88$ & $02 ' 11$ & 04 & $Q k$ & \\
\hline 011 & $818 \quad 3747499$ & STI & $17,13: 4 ?$ & $01: 51$ & 0.3 & $01:$ & \\
\hline 012 & 63 & FINE & $17,14: 14$ & $03^{\prime} 02$ & 01 & IK & \\
\hline 013 & G3 & STD & $17,14: 21$ & $03^{\prime} 08$ & 05 & $O K$ & \\
\hline 014 & IFPC FLIGHTLINK & FINE & $17.14: 59$ & 01,13 & 01 & $O K$ & \\
\hline 015 & 3013534690 & STD & $17.15: 01$ & $03 \cdot 04$ & 05 & $O K$ & \\
\hline 016 & 63 & STD & $17,15: 28$ & $05^{\prime} 40$ & 03 & OK & \\
\hline 017 & $510 \quad 246 \quad 6542$ & STD & $17,15: 35$ & $01 \cdot 37$ & 03 & $\mathrm{Or}$ & \\
\hline 018 & $1-602-964-9717$ & FINE & $17,16: 11$ & $04 \cdot 33$ & 01 & R. 1.4 & STOP \\
\hline 019 & $206 \quad 8720199$ & FINE & $17,17: 23$ & 01,34 & 02 & $O K$ & \\
\hline 020 & QLSEN - $702-431-8035$ & FIME & $17,22: 00$ & $02 \prime 11$ & 01 & $R . \overline{3} .3$ & \\
\hline 021 & $0 L S E N-702-431-8036$ & FINE & $17.22: 08$ & $01 \cdot 02$ & 01 & OK & \\
\hline 001 & G.3 & STO & $18,08: 03$ & $03 \cdot 09$ & 05 & DK & \\
\hline 002 & $704 \leq 823105$ & STD & $18,08: 54$ & $05 \cdot 45$ & 11 & DK & \\
\hline
\end{tabular}

